\title{
HANKEL MULTIPLIER TRANSFORMATIONS AND WEIGHTED $p$-NORMS ${ }^{(1)}$
}

\author{
BY \\ DOUGLAS L. GUY( $\left.{ }^{2}\right)$
}

Introduction. Let $L^{1}(-\infty, \infty)$ denote the Banach space of all real valued measurable functions $f$ on $(-\infty, \infty)$ such that

$$
\|f\|_{1}=(2 \pi)^{-1 / 2} \int_{-\infty}^{\infty}|f(x)| d x<\infty .
$$

Functions equal almost everywhere are identified. It is well known that if multiplication is given by the convolution

$$
[f * g](x)=(2 \pi)^{-1 / 2} \int_{-\infty}^{\infty} f(x-u) g(u) d u,
$$

then $L^{1}$ is a Banach algebra. Writing

$$
f^{\wedge}(y)=(2 \pi)^{-1 / 2} \int_{-\infty}^{\infty} f(x) e^{i y x} d x
$$

for the Fourier transform of $f(x)$, we have the familiar formula

$$
[f * g]^{\wedge}(y)=f^{\wedge}(y) g^{\wedge}(y)
$$

by Fubini's theorem.

Consider a transformation $T$ of functions on $(-\infty, \infty)$ to functions on $(-\infty, \infty)$ such that

$$
T[f * g]=T[f] * g=f * T[g] .
$$

In order to characterize such a transformation we apply (3) and obtain $T[f]^{\wedge} g^{\wedge}=f^{\wedge} T[g]^{\wedge}$. It follows that there is a function $\phi$ such that

$$
T[f]^{\wedge}(y)=\phi(y) f^{\wedge}(y) .
$$

If, on the other hand, (5) holds, then the transforms of the members of

Presented to the Society, November 25, 1955 under the title Marcinkiewicz theorem for Hankel transforms and December 29, 1955 under the title Weighted p-norms and Fourier transforms; received by the editors April 17, 1959.

(1) From the doctoral thesis submitted to the faculty of Washington University, St. Louis, Missouri, January, 1958. Research supported in part by Office of Scientific Research, U. S. Air Force.

(2) The author wishes to thank Professor I. I. Hirschman, Jr., for suggesting the problem and for his guidance in its solution. 
(4) are each equal to $\phi f^{\wedge} g^{\wedge}$. Since the Fourier transformation is one-one, (4) follows.

The inverse of the Fourier transformation is given by the formula

$$
f(x)=(2 \pi)^{-1 / 2} \int_{-\infty}^{\infty} f^{\wedge}(y) e^{-i x y} d y,
$$

it being necessary in general to interpret this integral by means of a summability process. With this in mind we write (5) in the form

$$
T f(x)=(2 \pi)^{-1 / 2} \int_{-\infty}^{\infty} \phi(y) f^{\wedge}(y) e^{-i x y} d y .
$$

The transformation $T$ is called the Fourier multiplier transformation given by $\phi$. Examples are furnished by the Riesz conjugate

$$
f^{\sim}(x)=\frac{1}{\pi} \int_{-\infty}^{\infty} f(u)(x-u)^{-1} d u,
$$

the partial Fourier integral

$$
S_{a}[f(x)]=(2 \pi)^{-1 / 2} \int_{-a}^{a} f^{\wedge}(y) e^{-i x y} d y,
$$

the translate

$$
T_{a}[f(x)]=f(x+a)
$$

and the derivative

$$
f^{\prime}(x)=d f / d x
$$

of $f$. These are given under suitable conditions by the functions $i$ sgn $y$, the characteristic function of the interval $[-a, a], e^{-i a y}$, and $-i y$ respectively.

A similar type of transformation may be defined using Fourier series instead of Fourier integrals. Suppose that $\left\{t_{n}\right\}$ is a given sequence of complex numbers and that a function $f$ defined on $[-\pi, \pi]$ has Fourier coefficients $a_{n}$. We write

$$
f(x) \sim \sum_{-\infty}^{\infty} a_{n} e^{i n x}
$$

and say that $T f(x)$ is the Fourier series multiplier transformation given by $\left\{t_{n}\right\}$ if

$$
T f(x) \sim \sum_{-\infty}^{\infty} t_{n} a_{n} e^{i n x} .
$$

J. Marcinkiewicz has proved the following important result [1939]: 
TheOREm. If $f \in L^{p}(-\pi, \pi), 1<p<\infty$, and if

(a)

$$
\left|t_{n}\right| \leqq C, \quad-\infty<n<\infty,
$$

and

$$
\sum_{ \pm 2^{n}+1}^{ \pm 2^{n+1}}\left|t_{k}-t_{k-1}\right| \leqq C, \quad 0<n<\infty,
$$

then

$$
\|T f\|_{p} \leqq A(p)\|f\| .
$$

This was generalized by I. I. Hirschman, Jr. [1955a, pp. 29-51 and p. 60] who replaced $L^{p}(-\pi, \pi)$ by the space $L^{\alpha, p}(-\pi, \pi)$ of all $f$ such that

$$
\|f\|_{\alpha, p}=\left[\int_{-\pi}^{\pi}|f(x)|^{p}|x|^{\alpha p} d x\right]^{1 / p}<\infty .
$$

These theorems were proved on the basis of some rather involved results of J. W. Littlewood and R. E. A. C. Paley [1937] as well as A. Zygmund $[1938 ; 1945]$. In the special case $p=2$, however, Hirschman obtained a proof which avoids these [1955a, pp. 51-60]. This proof is in addition applicable to certain orthogonal series other than Fourier series. Using this method Hirschman [1955b] obtained the result corresponding to Marcinkiewicz theorem for Legendre series multiplier transformations and weighted quadratic norms. In another paper Hirschman together with R. Askey [1959] extended this result to ultraspherical polynomials. Hirschman [1956b] has simplified this proof.

In the more difficult case $p \neq 2$ less is known. However important results have been obtained by H. Pollard [194:7; 1948], and [1949] for ultraspherical polynomials and by Hirschman [1957] for Jacobi polynomials. G. M. Wing has considered the case of Fourier-Bessel series [1950] and of Hankel transforms [1951]. Since the latter paper is of special interest to us, we consider its contents in a little more detail.

If $f \in L^{1}(0, \infty)$ and if $\nu \geqq-1 / 2$, we write

$$
F_{\nu}(y)=\int_{0}^{\infty} f(x) J_{\nu}(x y)(x y)^{1 / 2} d x
$$

for the Hankel transform of $f$ of order $\nu$. If $f \in L^{p}(0, \infty), 1<p \leqq 2$ and $1 / q$ $=1-1 / p$, then $F_{\nu}$ is given as the limit in the mean of order $q$ of partial integrals of (7). Writing

$$
S_{a}[f(x)]=\int_{0}^{a} F_{\nu}(y) J_{\nu}(x y)(x y)^{1 / 2} d y
$$

for the partial Hankel integral of $f$, Wing proved that 


$$
\left\|S_{a}[f]\right\|_{p} \leqq\|f\|_{p}
$$

and that $S_{a}[f]$ converges in the mean of order $p$ to $f$. Our main result here, the analogue of Marcinkiewicz theorem for Hankel multiplier transformations and weighted $p$-norms, includes this result of Wing.

We find it convenient to modify the definition (7) of the Hankel transform. We set

$$
d m_{\nu}(x)=\left[2^{\nu-1 / 2}(\nu+1 / 2)\right]^{-1} x^{2 \nu} d x
$$

where $\nu \geqq 0$. We write $H_{\nu}^{1}(0, \infty)$ for the Banach space of all real valued measurable functions $f$ such that

$$
\|f\|_{1}=\int_{0}^{\infty}|f(x)| d m_{\nu}(x)<\infty .
$$

We next set

$$
V_{\nu}(x)=2^{\nu-1 / 2}(\nu+1 / 2) J_{\nu-1 / 2}(x) x^{1 / 2-\nu}
$$

and call

$$
f^{\wedge}(y)=\int_{0}^{\infty} f(x) V_{\nu}(x y) d m_{\nu}(x)
$$

the (modified) Hankel transform. Using (7) and (11) we see that $f^{\wedge}$ is merely $y^{-\nu}$ times the ordinary Hankel transform of $x^{\nu} f(x)$ of order $\nu-1 / 2$.

We may introduce an operation of multiplication into $H_{\nu}^{1}$ in such a way that a Banach algebra results. Let $x, y$, and $z$ be non-negative real numbers and set $\Delta(x, y, z)$ be the area of the triangle with sides $x, y$, and $z$ if such a triangle exists and let it be zero if not. Then set

$$
D_{\nu}(x, y, z)=\frac{2^{3 \nu-5 / 2} \Gamma(\nu+1 / 2)^{2} \Delta(x, y, z)^{2 \nu-2}}{\Gamma(1 / 2) \Gamma(\nu)(x y z)^{2 \nu-1}} .
$$

As we shall show in Lemma $2 \mathrm{~A}$ below

$$
\int_{0}^{\infty} V_{\nu}(x t) D_{\nu}(x, y, z) d m_{\nu}(x)=V_{\nu}(y t) V_{\nu}(z t) .
$$

The convolution of two functions $f$ and $g$ in $H_{\nu}^{1}$ is defined by the formula

$$
\left[f^{*} g\right](x)=\int_{0}^{\infty} \int_{0}^{\infty} f(y) g(z) D_{\nu}(x, y, z) d m_{\nu}(y) d m_{\nu}(z) .
$$

Using (14) and Fubini's theorem, the relation

$$
\left[f^{*} g\right]^{\wedge}=f^{\wedge} g^{\wedge}
$$

follows easily. 
Since the transforms of $f *(g * h)$ and $(f * g) * h$ are each equal to $f^{\wedge} g^{\wedge} h^{\wedge}$ and since the modified Hankel transform is one-one, it follows that convolution is an associative operation. That it is commutative is immediate from (15) and Fubini's theorem. We next note that $V_{\nu}(0)=1$ and thus setting $t=0$ in (14) we have

$$
\int_{0}^{\infty} D_{\nu}(x, y, z) d m_{\nu}(x)=1
$$

for all $y$ and $z$. By Fubini's theorem we obtain

$$
\begin{aligned}
\|f * g\|_{1} & \leqq \int_{0}^{\infty} \int_{0}^{\infty} \int_{0}^{\infty}|f(y) g(z)| D_{\nu}(x, y, z) d m_{\nu}(y) d m_{\nu}(z) d m_{\nu}(x) \\
& =\int_{0}^{\infty}|f(y)| d m_{\nu}(y) \int_{0}^{\infty}|g(z)| d m_{\nu}(z) \int_{0}^{\infty} D_{\nu}(x, y, z) d m_{\nu}(x) \\
& =\|f\|_{1}\|g\|_{1} .
\end{aligned}
$$

We may now conclude that $H_{\nu}^{1}$ is a cornmutative Banach algebra.

If we consider a transformation $T$ of functions on $(0, \infty)$ into functions on $(0, \dot{\infty})$ such that

$$
T\left[f^{*} g\right]=T[f]^{*}=f^{*} T[g],
$$

it follows as in the case of Fourier Transforms that there is a function $\phi$ such that

$$
T[f]^{\wedge}(y)=\phi(y) f^{\wedge}(y)
$$

and conversely. We call $T$ the Hankel multiplier transformation given by $\phi$.

The construction above is based on results due to J. Delsarte [1938]. It may be of interest to summarize these here. We make no attempt at complete rigor.

Let $L_{x}$ be a linear operator on a set $A$ of functions. Suppose that $L_{x}$ has a continuous spectrum $S$ in the complex plane and that for each $\alpha \in S$ the corresponding eigenfunction is $j_{\alpha}$. Let these functions be normalized so that $j_{\alpha}(0)=1$ for each $\alpha$.

We next suppose that there is a sequence $\left\{\phi_{n}\right\}$ of functions in $A$ such that for each $\alpha$ in $S$ we have

$$
j_{\alpha}(x)=\sum_{0}^{\infty} \alpha^{n} \phi_{n}(x)
$$

Under these assumptions the generalized translation operator $T_{y}$ is given by the formula

$$
T_{y}[f(x)]=\sum_{0}^{\infty} \phi_{n}(y) L_{x}^{n}[f]
$$


Delsarte observed that $T_{y}[f(x)]$ is characterized as the solution $F(x, y)$ of the equation

$$
L_{x}[F(\cdot, y)]=L_{y}[F(x, \cdot)], \quad F(x, 0)=f(x) .
$$

He also noted that

$$
T_{\nu}\left[j_{\alpha}(x)\right]=j_{\alpha}(x) j_{\alpha}(y)
$$

For the first example let

$$
L_{x}[f]=f^{\prime}(x) .
$$

Then $S$ is the whole complex plane, $j_{\alpha}(x)=e^{\alpha x}$, and $\phi_{n}(x)=x^{n} / n$ !. Moreover equation (21) now reads

$$
F_{x}(x, y)=F_{y}(x, y), \quad F(x, 0)=f(x) .
$$

Taking Fourier transforms of both sides, we obtain

$$
-i t F^{\wedge}(t, y)=F_{y}^{\wedge}(t, y), \quad F^{\wedge}(t, 0)=f^{\wedge}(t) .
$$

This ordinary equation has the solution

$$
F^{\wedge}(t, y)=f^{\wedge}(t) e^{-i t y} .
$$

Taking the inverse Fourier transform, we conclude that

$$
T_{y}[f(x)]=f(x+y) .
$$

Thus $T_{y}$ is the ordinary translation operator and formula (20) is Taylor's formula. Equation (22) is the familiar relation $e^{\alpha(x+y)}=e^{\alpha x} e^{\alpha y}$.

For the second example let

$$
L_{x}[f]=f^{\prime \prime}(x)+\frac{2 \nu}{x} f^{\prime}(x) .
$$

By standard formulas from the theory of Bessel functions, Sneddon [1951, pp. 511-512], we see that

$$
L_{x}\left[V_{\nu}(a x)\right]=-a^{2} V_{\nu}(a x) .
$$

It follows that

$$
j_{\alpha}(x)=V_{\nu}\left(i \alpha^{1 / 2} x\right) .
$$

Moreover, equation (21) now reads

$$
F_{x x}(x, y)+\frac{2 \nu}{x} F_{x}(x, y)=F_{y y}(x, y)+\frac{2 \nu}{y} F_{y}(x, y), \quad F(x, 0)=f(x) .
$$

Taking modified Hankel transforms, we have 


$$
-t^{2} F^{\wedge}(t, y)=\hat{F_{y y}}(t, y)+\frac{2 \nu}{y} \hat{F_{y}}(t, y), \quad F^{\wedge}(t, 0)=f^{\wedge}(t)
$$

by a known formula for the Hankel transform of $L_{x}[F]$, Sneddon [1951, p. 61]. This modified Bessel equation has the solution

$$
F^{\wedge}(t, y)=f^{\wedge}(t) V_{\nu}(t y) .
$$

Taking transforms again, we conclude that

$$
\begin{aligned}
F(x, y) & =T_{\nu}[f(x)] \\
& =\int_{0}^{\infty} V_{\nu}(t y) V_{\nu}(t x) d m_{\nu}(t) \int_{0}^{\infty} f(z) V_{\nu}(z t) d m_{\nu}(z) \\
& =\int_{0}^{\infty} f(z) D_{\nu}(x, y, z) d m_{\nu}(z)
\end{aligned}
$$

using (14) and inversion of the order of integration $\left({ }^{3}\right)$. The formula (15) for the convolution now reads

$$
\left[f^{*} g\right](x)=\int_{0}^{\infty} T_{y}[f(x)] g(y) d m_{\nu}(y) .
$$

This corresponds to formula (1) for the ordinary convolution.

We now see a rather detailed analogy between $H_{\nu}^{1}$ and $L^{1}$. That this analogy goes even deeper is shown by the following easily established result:

THEOREM. If $\nu \geqq 0$, then $H_{\nu}^{1}(0, \infty)$ is a regular commutative semi-simple Banach algebra and the space of closed maximal ideals is isomorphic to the set $[0, \infty]$ endowed with the usual topology.

The ultraspherical polynomials also given rise to Banach algebras. See I. I. Hirschman [1956b]. In this connection see also B. M. Levitan [1949] and S. Bochner [1954].

In Part I below we shall be concerned with Hankel multiplier transformations defined on the Banach space $H_{\nu}^{\alpha, 2}(0, \infty)$ of all real valued measurable functions $f$ such that

$$
\|f\|_{\alpha, 2}=\left[\int_{0}^{\infty} f(x)^{2} x^{2 \alpha} d m_{\nu}(x)\right]^{1 / 2}<\infty .
$$

Using the direct method of Hirschman we prove the analogue of Marcinkiewicz theorem for Hankel multiplier transformations.

In Part II the same result is proved for weighted p-norms. The method entails proving first the corresponding result for Fourier multiplier trans-

(3) Delsarte gives the solution without using transforms. His solution may be converted to ours by an easy change of variables. 
formations. The following lemma, which may be of some interest in itself, is then employed to carry this result over to Hankel multiplier transformations:

Lemma. If $\int_{0}^{\infty} s^{-1 / p-\alpha}|g(s)| d s<\infty$ and $g$ has ordinary Hankel transforms $G_{\nu}$ and $G_{\mu}$ lying in $L^{\alpha, p}(0, \infty), 1<p<\infty,-1 / p<\alpha<1-1 / p, \nu, \mu>-1 / 2$, then $\left\|G_{\nu}\right\|_{\alpha, p} /\left\|G_{\mu}\right\|_{\alpha, p}$ lies between positive constants.

\section{PART I. WEIGHTED QUADRATIC NORMS}

1. Preliminary results. We begin by proving some general results about the spaces $H_{\nu}^{\alpha, 2}(0, \infty)$ and $L^{\alpha, 2}(0, \infty)$. We note that $f$ is in the former if and only if $f(x) x^{\nu}$ is in the latter. If $\lim _{a \rightarrow \infty}\left\|f_{a}-f\right\|_{\alpha, 2}=0$, we say that $f_{a}$ converges to $f$ in $L^{\alpha, 2}$ or $H_{\nu}^{\alpha, 2}$ and write

$$
\underset{a \rightarrow \infty}{\lim .{ }^{\alpha, 2}} f_{a}=f .
$$

Writing $H_{\nu}^{2}$ for $H_{\nu}^{0,2}$, we note that if $f \in H_{\nu}^{2}$, then $x^{\nu} f(x) \in L^{2}$. The ordinary Hankel transform $F_{\nu-1 / 2}$ of order $\nu-1 / 2$ of this latter function is given as a limit in the mean of order two of partial integrals

$$
F_{\nu-1 / 2}(y, a)=\int_{0}^{a} x^{\nu} f(x) J_{\nu-1 / 2}(x y)(x y)^{1 / 2} d x .
$$

Setting $f^{\wedge}(y)=y^{-\nu} F_{v-1 / 2}(y)$, we see from formulas (9), (11), (12) and (23) of the introduction that $f^{\wedge}(y)$ is the limit in $H_{\nu}^{2}$ of partial integrals

$$
f^{\wedge}(y, a)=\int_{0}^{a} f(x) V_{\nu}(x y) d m_{\nu}(x) .
$$

It is now evident that each of the following results is an immediate consequence of the corresponding result for ordinary Hankel transforms in $L^{2}$, Titchmarsh [1922, p. 473]:

THEOREM 1A. If $f \in H_{\nu}^{2}(0, \infty), \nu \geqq 0$, then the (modified) Hankel transform $f^{\wedge}$ of $f$ of order $\nu$ exists as a limit in $H_{\nu}^{2}$ of partial integrals (3) as $a \rightarrow \infty$. Moreover the following hold:

(a) Parseval's theorem,

$$
\left\|f^{\wedge}\right\|_{2}=\|f\|_{2}
$$

(b) the inversion formula

$$
\left[f^{\wedge}\right]^{\wedge}=f
$$

(c) the uniqueness theorem,

$$
\text { if } f^{\wedge}(y)=0 \text { for all } y \text {, then } f(x)=0 \text { a.e., }
$$

and if $g$ is also in $H_{\nu}^{2}$, 
(d) Plancherel's theorem,

$$
\int_{0}^{\infty} f(x) g(x) d m_{\nu}(x)=\int_{0}^{\infty} f^{\wedge}(y) g^{\wedge}(y) d m_{\nu}(y) .
$$

In case $\alpha \neq 2$, such powerful results ard not obtainable. However we prove the following:

THEOREM 1B. If $f \in H_{\nu}^{\alpha, 2}(0, \infty), 0 \leqq \alpha<1 / 2, \nu \geqq 0$, and $\beta=-\alpha$, then the (modified) Hankel transform $f^{\wedge}(y)$ exists as a limit in $H_{\nu}^{\beta, 2}(0, \infty)$ of partial integrals

$$
\hat{f_{a}}(y)=\int_{a^{-1}}^{a} f(x) V_{\nu}(x y) d m_{\nu}(x)
$$

as $a \rightarrow \infty$. In addition we have

$$
\left\|f^{\wedge}\right\|_{\beta, 2} \leqq A(\alpha, \nu)\|f\|_{\alpha, 2}
$$

This is a consequence of a more general result.

Theorem 1B'. Suppose that $k(x, y)$ is bounded for $x, y \geqq 0$ and that a bounded transformation of $L^{2}(0, \infty)$ into itself is given by

$$
f(x) \rightarrow F(y)=\operatorname{lim.m}_{a \rightarrow \infty}^{2} \int_{0}^{a} f(x) k(x, y) d x .
$$

Then for every $f \in L^{\alpha, 2}(0, \infty), 0 \leqq \alpha<1 / 2$, a function $F$ exists as a limit in $L^{\beta, 2}(0, \infty), \beta=-\alpha$, of partial integrals

$$
F_{a}(y)=\int_{a^{-1}}^{a} f(x) k(x y) d x
$$

as $a \rightarrow \infty$. In addition we have

$$
\|F\|_{\beta, 2} \leqq A(\alpha, k)\|f\|_{\alpha, 2} .
$$

It can be shown that this result is in turn a consequence of a theorem of R. E. A. C. Paley [1931]. However, it is just as easy to prove it directly. This latter proof, which we now give, is based on ideas of Paley [1931] and Hirschman [1956a]. We use $A(\alpha, \cdots)$ to denote a constant depending on $\alpha, \cdots$, and not necessarily having the same value at every occurrence.

Proof. The case $\alpha=0$ is trivial. Since $A(\alpha, k)$ is logarithmically convex as a function $\alpha\left({ }^{4}\right)$, it is enough to prove the theorem for the range $1 / 4<\alpha<1 / 2$.

We first establish two inequalities. Let

$$
K_{n}(y)=\int_{2^{n}}^{2^{n+1}} f(x) k(x, y) d x, \quad-\infty<n<\infty
$$

and

(4) See Hirschman [1956a]. 


$$
W_{n}^{2}=\int_{2^{n}}^{2^{n+1}} f(x)^{2} x^{2 \alpha} d x, \quad-\infty<n<\infty .
$$

By the boundedness of $k$ and the Schwarz inequality we have

$$
\begin{aligned}
& \underset{0 \leqq y<\infty}{\text { l.u.b. }}\left|K_{n}(y)\right| \leqq A \int_{2^{n}}^{2^{n+1}}|f(x)| d x \\
& \leqq A\left[\int_{2^{n}}^{2^{n+1}} f(x)^{2} x^{2 \alpha} d x\right]^{1 / 2}\left[\int_{2^{n}}^{2^{n+1}} x^{-2 \alpha} d x\right]^{1 / 2} \\
& \leqq A W_{n} 2^{n / 2-\alpha n} \text {. }
\end{aligned}
$$

Since we have a bounded transformation of $L^{2}$ into itself, it follows that

$$
\begin{aligned}
\left\|K_{n}\right\|_{2} & \leqq A\left[\int_{2^{n}}^{2^{n+1}} f(x)^{2} d x\right]^{1 / 2} \\
& \leqq A\left[\begin{array}{c}
\text { l.u.b. } \\
2^{n}<x<2^{n+1}
\end{array} x^{-2 \alpha}\right]^{1 / 2}\left[\int_{2^{n}}^{2^{n+1}} f(x)^{2} x^{2 \alpha} d x\right]^{1 / 2} \\
& =A W_{n} 2^{-\alpha n} .
\end{aligned}
$$

Now suppose that $a>0$ and set

$$
I_{n, m}=\left(\int_{0}^{a}+\int_{a}^{\infty}\right)\left|K_{n}(y) K_{m}(y)\right| y^{-2 \alpha} d y=I_{n, m}^{\prime}+I_{n, m}^{\prime \prime} .
$$

Then, supposing for the moment that $n \leqq m$, we have by (8)

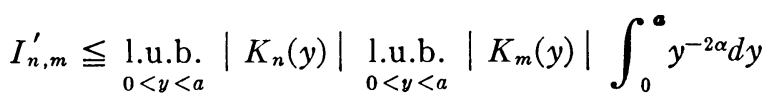

$$
\begin{aligned}
& \leqq A W_{n} W_{m} 2^{-\alpha n+n / 2-\alpha m+m / 2} a^{1-2 \alpha}
\end{aligned}
$$

and by (9) as well

$$
\begin{aligned}
& I_{n, m}^{\prime \prime} \leqq \underset{a<y<\infty}{\text { l.u.b. }}\left|K_{n}(y)\right|\left[\int_{a}^{\infty} K_{m}(y)^{2} d y\right]^{1 / 2}\left[\int_{a}^{\infty} y^{-4 \alpha} d y\right]^{1 / 2} \\
& \leqq A W_{n} W_{m} 2^{-\alpha n+n / 2-\alpha m} a^{1 / 2-2 \alpha} \text {. }
\end{aligned}
$$

Setting $a=2^{-m}$, we obtain

$$
I_{n, m} \leqq A W_{n} W_{m} 2^{-(1 / 2-\alpha)|m-n|} .
$$

Now let

$$
F_{M}(y)=\int_{2^{-M}}^{2^{M+1}} f(x) k(x, y) d x=\sum_{-M}^{M} K_{n}(y), \quad M=1,2, \cdots
$$

Then, using the estimate 


$$
2|a b| \leqq a^{2}+b^{2}
$$

we obtain

$$
\begin{aligned}
\left\|F_{M}\right\|_{\beta, 2}^{2} & \leqq \sum_{n, m=-M}^{M} I_{n, m} \\
& \leqq A \sum_{n, m=-M}^{M} W_{n} W_{m} 2^{-(1 / 2-\alpha)|m-n|} \\
& \leqq A \sum_{n=-M}^{M} W_{n}^{2} \sum_{m=-M}^{M} 2^{-(1 / 2-\alpha)|m-n|} \\
& \leqq A \sum_{n=-M}^{M} W_{n}^{2} \leqq A\|f\|_{\alpha, 2}^{2} .
\end{aligned}
$$

Since the last term is independent of $M$, we are done.

The kernel $J_{\nu-1 / 2}(x y)(x y)^{1 / 2}$ satisfies the hypotheses of Theorem $1 \mathrm{~B}^{\prime}$; that is, we have the result for the ordinary Hankel transform. By the relation of the modified Hankel transform to this, we have Theorem 1B.

By standard methods, A. Zygmund [1935, pp. 208-211], Theorem 1B' may be somewhat strengthened.

THEOREM 1C. If, under the hypotheses of Theorem $1 \mathrm{~B}^{\prime}, F^{*}$ is a nonincreasing function equimeasurable with $F$, then

$$
\left\|F^{*}\right\|_{\beta, 2} \leqq A(\alpha, k)\|f\|_{\alpha, 2} .
$$

As an easy consequence we also have the following:

Corollary 1D. If $f \in H_{\nu}^{\alpha, 2}(0, \infty), 0<\alpha<1 / 2, \nu \geqq 0$, and if $0<a<\infty$, then

$$
\int_{0}^{a} f^{\wedge}(y)^{2}(a-y)^{-2 \alpha} d m_{\nu}(y) \leqq A(\alpha, \nu)\|f\|_{\alpha, 2}^{2}
$$

and

$$
\int_{a}^{\infty} f^{\wedge}(y)^{2}(y-a)^{-2 \alpha} d m_{\nu}(y) \leqq A(\alpha, \nu)\|f\|_{\alpha, 2}^{2} .
$$

2. The basic norm relation. In this section we derive the key result used in the proof of the main theorem. More explicitly, we find a function $S_{\alpha}(x, y)$ such that for each $f$ in $H_{\nu}^{\alpha, 2}, 0<\alpha<1 / 2$, the basic norm relation

$$
\|f\|_{\alpha, 2}^{2}=\frac{1}{2} \int_{0}^{\infty} \int_{0}^{\infty}\left[f^{\wedge}(y)-f^{\wedge}(z)\right]^{2} S_{\alpha}(x, y) d m_{\nu}(y) d m_{\nu}(z)
$$

holds.

We recall formula (13) from the introduction and prove the following: 
Lemma 2A. If $\nu>0$ and $x, y, z, t \geqq 0$, then

$$
\int_{0}^{\infty} V_{\nu}(z t) D_{\nu}(x, y, z) d m_{\nu}(z)=V_{\nu}(x t) V_{\nu}(y t) .
$$

Proof. By Watson [1944, p. 411]

$$
\int_{0}^{\infty} J_{p}(x t) J_{p}(y t) J_{p}(z t) t^{1-p} d t=\frac{2^{p-1} \Delta(x, y, z)^{2 p-1}}{\Gamma(p+1 / 2) \Gamma(1 / 2)(x y z)^{p}}
$$

when $p>-1 / 2$. Setting $p=\nu-1 / 2$ and multiplying both sides by $2^{2 \nu-1}(x y z)^{1 / 2-\nu} \Gamma(\nu+1 / 2)^{2}$, we have

$$
\left[V_{\nu}(x t) V_{\nu}(y t)\right]^{\wedge}(z)=D_{\nu}(x, y, z) .
$$

An application of MacRobert's version of the Hankel inversion formula for analytic functions, I. N. Sneddon [1951, p. 53], completes the proof.

Suppose now that $\phi$ is a non-negative measurable function such that the integral

$$
s(x)=\int_{0}^{\infty}\left[1-V_{\nu}(x t)\right] \phi(t) d t
$$

is finite for all $x \geqq 0$. Since $\left|V_{\nu}(x t)\right| \leqq 1, s(x) \geqq 0$. However, it does not follow that $\phi \in L^{1}$, because $V_{\nu}(0)=1$. Nevertheless, when $\phi \in L^{1}, s(x)$ is bounded and $s(0)=0$. In any case, $s(x) \rightarrow 0$ as $x \rightarrow 0$ by the dominated convergence theorem. We set

$$
S(y, z)=\int_{0}^{\infty} \phi(t) D_{\nu}(t, y, z) d t
$$

Since $D_{\nu}(t, y, z) \geqq 0, S(y, z) \geqq 0$.

Lemma 2B. If $\phi, s$, and $S$ are defined as above and if $f \in H_{\nu}^{2}, \nu>0$, then

$$
\int_{0}^{\infty} f(x)^{2} s(x) d m_{\nu}(x)=\frac{1}{2} \int_{0}^{\infty} \int_{0}^{\infty}\left[f^{\wedge}(y)-f^{\wedge}(z)\right]^{2} S(y, z) d m_{\nu}(y) d m_{\nu}(z) .
$$

Proof. We first suppose that

$$
p=\int_{0}^{\infty} \phi(t) d t<\infty
$$

and

$$
f \in H_{\nu}^{1} \cap H_{\nu}^{2}
$$


Then we obtain for the double integral $I$ on the right side above

$$
\begin{aligned}
I= & \frac{1}{2} \int_{0}^{\infty} \int_{0}^{\infty} f^{\wedge}(y)^{2} S(y, z) d m_{\nu}(y) d m_{\nu}(z) \\
& +\frac{1}{2} \int_{0}^{\infty} \int_{0}^{\infty} f^{\wedge}(z)^{2} S(y, z) d m_{\nu}(y) d m_{\nu}(z) \\
& -\int_{0}^{\infty} \int_{0}^{\infty} f^{\wedge}(y) f^{\wedge}(z) S(y, z) d m_{\nu}(y) d m_{\nu}(z) \\
= & I_{1}+I_{2}+I_{3} .
\end{aligned}
$$

We shall show presently that the last three integrals are finite. We have

$$
\begin{aligned}
I_{1} & =\frac{1}{2} \int_{0}^{\infty} f^{\wedge}(y)^{2} d m_{\nu}(y) \int_{0}^{\infty} S(y, z) d m_{\nu}(z) \\
& =\frac{1}{2} \int_{0}^{\infty} f^{\wedge}(y)^{2} d m_{\nu}(y) \int_{0}^{\infty} d m_{\nu}(z)=\int_{0}^{\infty} \phi(u) D_{\nu}(u, y, z) d u \\
& =\frac{1}{2} \int_{0}^{\infty} f^{\wedge}(y)^{2} d m_{\nu}(y) \int_{0}^{\infty} \phi(u) d u \int_{0}^{\infty} D_{\nu}(u, y, z) d m_{\nu}(z) .
\end{aligned}
$$

Setting $t=0$ in Lemma $2 \mathrm{~A}$ we have

$$
\int_{0}^{\infty} D_{\nu}(u, y, z) d m_{\nu}(z)=1
$$

for all $u$ and $y$. Thus $D_{\nu} \in H_{\nu}^{1}$ as a function of $z$. It follows that $S(y, z) \in H_{\nu}^{1}$ as a function of $z$. Moreover we now have

$$
I_{1}=\frac{1}{2} p \int_{0}^{\infty} f^{\wedge}(y)^{2} d m_{\nu}(y)=\frac{1}{2} p \int_{0}^{\infty} f(x)^{2} d m_{\nu}(x)
$$

by Parseval's theorem. Similarly we obtain

$$
I_{1}=\frac{1}{2} p \int_{0}^{\infty} f(x)^{2} d m_{\nu}(x) .
$$

Using the estimate $2|a b| \leqq a^{2}+b^{2}$, we see that $I_{3}$ converges absolutely in view of (5) and (6). Thus we may apply Fubini's theorem to $I_{3}$, obtaining

$$
\begin{aligned}
I_{3} & =-\int_{0}^{\infty} f^{\wedge}(y) d m_{\nu}(y) \int_{0}^{\infty} f^{\wedge}(z) S(y, z) d m_{\nu}(z) \\
& =-\int_{0}^{\infty} f^{\wedge}(y) d m_{\nu}(y) \int_{0}^{\infty} S(y, z) d m_{\nu}(z) \int_{0}^{\infty} f(x) V_{\nu}(z x) d m_{\nu}(x) .
\end{aligned}
$$

Since $f \in H_{v}^{1}, S \in H_{\nu}^{1}$ as a function of $z$, and $V_{v}$ is bounded, we have 


$$
\begin{aligned}
\int_{0}^{\infty} & S(y, z) d m_{\nu}(z) \int_{0}^{\infty} f(x) V_{\nu}(z x) d m_{\nu}(x) \\
& =\int_{0}^{\infty} f(x) d m_{\nu}(x) \int_{0}^{\infty} S(y, z) V_{\nu}(z x) d m_{\nu}(z) \\
& =\int_{0}^{\infty} f(x) d m_{\nu}(x) \int_{0}^{\infty} V_{\nu}(z x) d m_{\nu}(z) \int_{0}^{\infty} D_{\nu}(u, y, z) \phi(u) d u .
\end{aligned}
$$

Since $\phi \in L^{1}, D_{\nu} \in H_{\nu}^{1}$ as a function of $z$, and $V_{\nu}$ is bounded, we have by Fubini's theorem and Lemma $2 \mathrm{~A}$

$$
\begin{aligned}
\int_{0}^{\infty} & V_{\nu}(z x) d m_{\nu}(z) \int_{0}^{\infty} \phi(u) D_{\nu}(u, y, z) d u \\
& =\int_{0}^{\infty} \phi(u) d u \int_{0}^{\infty} D_{\nu}(u, y, z) V_{\nu}(z x) d m_{\nu}(z) \\
& =V_{\nu}(y x)[p-s(x)] .
\end{aligned}
$$

Inserting this in (8) and the result in (7), we see that

$$
\begin{aligned}
I_{3} & =\int_{0}^{\infty} f^{\wedge}(y) d m_{\nu}(y) \int_{0}^{\infty} f(x)[s(x)-p] V_{\nu}(x y) d m_{\nu}(x) \\
& =\int_{0}^{\infty} f^{\wedge}(y)[f(x)[s(x)-p]]^{\wedge}(y) d m_{\nu}(y) \\
& =\int_{0}^{\infty} f(x)^{2}[s(x)-p] d m_{\nu}(x) .
\end{aligned}
$$

Combining (4), (5), (6), and (9), we have the theorem under the restrictions (a) and (b).

We next suppose that $f$ is any function in $H_{\nu}^{1}$ and remove restriction (b). By (a), $s(x)$ is bounded and thus

$$
\int_{0}^{\infty} f(x)^{2} s(x) d m_{\nu}(x)<\infty .
$$

We set $f_{a}(x)$ equal to $f(x)$ if $x \in\left[a^{-1}, a\right]$ and equal to zero otherwise. Then $\hat{f_{a}} \rightarrow \hat{f^{\wedge}}$ in $H_{\nu}^{2}$. Thus there is an increasing sequence $\left\{a_{n}\right\}$ such that $a_{n} \rightarrow \infty$ and $\hat{a_{n}}(y) \rightarrow f^{\wedge}(y)$ pointwise as $n \rightarrow \infty$. Since $f_{a_{n}} \in H_{\nu}^{1} \cap H_{\nu}^{2}$, we have

$$
\int_{0}^{\infty} f_{a_{n}}(x)^{2} s(x) d m_{\nu}(x)=\frac{1}{2} \int_{0}^{\infty} \int_{0}^{\infty}\left[\hat{a_{n}}(y)-\hat{f_{a_{n}}}(z)\right]^{2} S(y, z) d m_{\nu}(y) d m_{\nu}(z) .
$$

We now have, by Fatou's lemma, 


$$
\int_{0}^{\infty} f(x)^{2} s(x) d m_{\nu}(x) \geqq \frac{1}{2} \int_{0}^{\infty} \int_{0}^{\infty}\left[f^{\wedge}(y)-f^{\wedge}(z)\right]^{2} S(y, z) d m_{\nu}(y) d m_{\nu}(z) .
$$

In order to prove the reverse inequality we write

$$
f_{a}^{\wedge}(y)-f_{a}(z)=\left[\left(f_{a}^{\wedge}(y)-f^{\wedge}(y)\right)-\left(f_{\hat{a}}^{\wedge}(z)-f^{\wedge}(z)\right)\right]+\left[f^{\wedge}(y)-f^{\wedge}(z)\right] .
$$

Using the estimate

$$
(a-b)^{2} \leqq\left(1+e^{\alpha}\right) a^{2}+\left(1+e^{-\alpha}\right) b^{2},
$$

we see that

$$
\begin{aligned}
{\left[f_{\hat{a}}^{\hat{a}}(y)-f_{\hat{a}}^{\wedge}(z)\right]^{2} \leqq } & \left(1+e^{\alpha}\right)\left[\left(f_{\hat{a}}^{\hat{a}}(y)-f^{\wedge}(y)\right)-\left(f_{\hat{a}}^{\hat{(}}(z)-f^{\wedge}(z)\right)\right] \\
& +\left(1+e^{-\alpha}\right)\left[f^{\wedge}(y)-f^{\wedge}(z)\right]^{2} .
\end{aligned}
$$

Therefore we have

$$
\begin{aligned}
& \frac{1}{2}\left(1+e^{-\alpha}\right) \int_{0}^{\infty} \int_{0}^{\infty}\left[f^{\wedge}(y)-f^{\wedge}(z)\right]^{2} S(y, z) d m_{\nu}(y) d m_{\nu}(z) \\
& \quad \geqq \frac{1}{2} \int_{0}^{\infty} \int_{0}^{\infty}\left[f_{a}^{\wedge}(y)-f_{a}^{\wedge}(z)\right]^{2} S(y, z) d m_{\nu}(y) d m_{\nu}(z) \\
& \quad-\frac{1}{2}\left(1+e^{\alpha}\right) \int_{0}^{\infty} \int_{0}^{\infty}\left[\left(f_{\hat{a}}^{\hat{a}}(y)-f^{\wedge}(y)\right)-\left(f_{\hat{a}}^{\wedge}(z)-f^{\wedge}(z)\right)\right]^{2}
\end{aligned}
$$

$\cdot S(y, z) d m_{\nu}(y) d m_{\nu}(z)$.

Since $f_{a} \in H_{\nu}^{1} \cap H_{\nu}^{2}$,

$$
\frac{1}{2} \int_{0}^{\infty} \int_{0}^{\infty}\left[f_{a}^{\wedge}(y)-f_{a}^{\hat{a}}(z)\right]^{2} S(y, z) d m_{\nu}(y) d m_{\nu}(z)=\int_{0}^{\infty} f_{\hat{a}}^{\hat{}}(x)^{2} s(x) d m_{\nu}(x) .
$$

By (11) we also have

$$
\begin{aligned}
\frac{1}{2} \int_{0}^{\infty} \int_{0}^{\infty}\left[\left(f_{\hat{a}}^{\hat{y}}(y)-f^{\wedge}(y)\right)-\left(f_{\hat{a}}(z)-f^{\wedge}(z)\right)\right]^{2} S(y, z) d m_{\nu}(y) d m_{\nu}(z) \\
\\
\leqq \int_{0}^{\infty}\left[f_{a}(x)-f(x)\right]^{2} s(x) d m_{\nu}(x) .
\end{aligned}
$$

Combining the last three expressions and letting $a \rightarrow \infty$, we obtain

$$
\frac{1}{2}\left(1+e^{-\alpha}\right) \int_{0}^{\infty} \int_{0}^{\infty}\left[f^{\wedge}(y)-f^{\wedge}(z)\right]^{2} S(y, z) d m_{\nu}(y) d m_{\nu}(z) \geqq \int_{0}^{\infty} f(x)^{2} s(x) d m_{\nu}(x) .
$$

Next letting $\alpha \rightarrow \infty$, we conclude that

$$
\frac{1}{2} \int_{0}^{\infty} \int_{0}^{\infty}\left[f^{\wedge}(y)-f^{\wedge}(z)\right]^{2} S(y, z) d m_{\nu}(y) d m_{\nu}(z) \geqq \int_{0}^{\infty} f(x)^{2} s(x) d m_{\nu}(x) .
$$


The removal of the restriction (a) is all that remains. Suppose that $\int_{0}^{\infty} \phi(t) d t=\infty$. It follows that there is a nondecreasing sequence of non-negative functions $\phi_{n}$ in $L^{1}$ such that $\phi_{n}(t) \rightarrow \phi(t)$ a.e. With evident notations

$$
\int_{0}^{\infty} f(x)^{2} s_{n}(x) d m_{\nu}(x)=\frac{1}{2} \int_{0}^{\infty} \int_{0}^{\infty}\left[f^{\wedge}(y)-f^{\wedge}(z)\right]^{2} S_{n}(x, y) d m_{\nu}(y) d m_{\nu}(z) .
$$

By (2) and (3) we see that $\left\{s_{n}\right\}$ and $\left\{S_{n}\right\}$ are nondecreasing which converge to $s$ and $S$ respectively. Thus we may obtain the desired result by letting $n \rightarrow \infty$ in (13).

Theorem 2C. If $f \in H_{\nu}^{\alpha, 2}, 0<\alpha<1 / 2, \nu>0$, then

$$
\|f\|_{\alpha, 2}^{2}=\frac{1}{2} \int_{0}^{\infty} \int_{0}^{\infty}\left[f^{\wedge}(y)-f^{\wedge}(z)\right]^{2} S_{\alpha}(y, z) d m_{\nu}(y) d m_{\nu}(z)
$$

where

$$
S_{\alpha}(y, z)=\int_{0}^{\infty} t^{-1-2 \alpha} D_{\nu}(t, y, z) d t / \int_{0}^{\infty} t^{-1-2 \alpha}\left[1-V_{\nu}(t)\right] d t .
$$

Proof. Let $c=\int_{0}^{\infty} t^{-1-2 \alpha}\left[1-V_{\nu}(t)\right] d t$. Since $1-V_{\nu}(t)=O\left(t^{2}\right)$ as $t \rightarrow 0, c$ is finite. It is also positive. Setting $\phi(t)=c^{-1} t^{-1-2 \alpha}$ in Lemma $2 \mathrm{~B}$, we see that

$$
\begin{aligned}
s(x) & =c^{-1} \int_{0}^{\infty} t^{-1-2 \alpha}\left[1-V_{\nu}(x t)\right] d t \\
& =c^{-1} \int_{0}^{\infty} x^{2 \alpha} u^{-1-2 \alpha}\left[1-V_{\nu}(u)\right] d u=x^{2 \alpha} .
\end{aligned}
$$

The theorem is now proved for all $f \in H_{\nu}^{2} \cap H_{\nu}^{\alpha, 2}$.

Suppose next that $f$ is any function in $H_{\nu}^{\alpha, 2}$ and let $f_{a}$ be defined as in the proof of Lemma 2B. Since $f_{a} \in H_{\nu}^{2} \cap H_{\nu}^{\alpha, 2}$, we have

$$
\left\|f_{a}\right\|_{\alpha, 2}^{2}=\frac{1}{2} \int_{0}^{\infty} \int_{0}^{\infty}\left[\hat{f_{a}}(y)-f_{\hat{a}}(z)\right]^{2} S_{\alpha}(y, z) d m_{\nu}(y) d m_{\nu}(z) .
$$

Because $f_{a} \rightarrow f$ in $H_{\nu}^{\alpha, 2}, \hat{f_{a}} \rightarrow f^{\wedge}$ in $H_{\nu}^{-\alpha, 2}$ by Theorem 1B. It follows that there is a nondecreasing sequence $\left\{a_{n}\right\}$ such that $\hat{a_{n}} \rightarrow f^{\wedge}$ pointwise a.e. The steps of the last half of the proof of Lemma $2 \mathrm{~B}$ may now be repeated to complete the proof of this theorem.

Corollary 2D. If $f$ and $g$ are in $H_{\nu}^{\alpha, 2}, 0<\alpha<1 / 2, \nu>0$, then

$$
\begin{aligned}
& \int_{0}^{\infty} f(x) g(x) x^{2 \alpha} d m_{\nu}(x) \\
& =\frac{1}{2} \int_{0}^{\infty} \int_{0}^{\infty}\left[f^{\wedge}(y)-f^{\wedge}(z)\right]\left[g^{\wedge}(y)-g^{\wedge}(z)\right] S_{\alpha}(y, z) d m_{\nu}(y) d m_{\nu}(z) .
\end{aligned}
$$


3. The main theorem. We say that a transformation $T$ of $H_{v}^{\alpha, 2}$ into itself is the Hankel multiplier transformation given by a function $\phi$ if

$$
T[f]^{\wedge}(y)=\phi(y) f^{\wedge}(y) \quad \text { a.e. }
$$

for every $f$ in the dense subspace $H_{\nu}^{2} \cap H_{\nu}^{\alpha, 2}$. In this section we find a condition on $\phi$ sufficient to assure that $T$ is a bounded transformation.

We note that if $\phi$ is bounded and $f \in H_{\nu}^{2} \cap H_{\nu}^{\alpha, 2}$, then $\phi f^{\wedge} \in H_{\nu}^{2}$. If we set $T f=\left[\phi f^{\wedge}\right]^{\wedge}$, we have (1) by the inversion formula. If in addition we prove that $T$ is bounded on $H_{\nu}^{2} \cap H_{\nu}^{\alpha, 2}$, then, by a well known theorem, $T$ has a unique extension to a bounded linear transformation on $H_{\nu}^{\alpha, 2}$. See Theorem $3 \mathrm{E}$ below. We first prove some lemmas.

Lemma 3A. If $0<\alpha<1 / 2$ and $\nu>0$, then we have

$$
S_{\alpha}(y, z) \leqq A(\alpha, \nu)|y-z|^{-1-2 \alpha}(y+z)^{-2 \nu}
$$

for all $y$ and $z \geqq 0$.

Proof. By Theorem 3C we have by the definition of $D_{v}$

$$
S_{\alpha}(y, z)=A(\nu)(y z)^{1-2 \alpha} \int_{|y-z|}^{y+z} t^{-2 \alpha-2 \nu} \Delta(y, z, t)^{2 \nu-2} d t .
$$

By Hero's formula

$$
4 \Delta(y, z, t)=[(y+z+t)(y+z-t)(y-z+t)(z-y+t)]^{1 / 2}
$$

and therefore we may write $S_{\alpha}(y, z)$ in the form

$$
A(\nu)(y z)^{1-2 \nu} \int_{|y-z|}^{y+z}[(y+z+t)(y+z-t)(y-z+t)(z-y+t)]^{\nu-1} t^{-2 \alpha-2 v} d t .
$$

We next consider two cases according as $y>z$ or $z>y$. By symmetry we need consider only one of these. Of course the case $y=z$ is trivial. Let us suppose then that $y>z$.

If in addition $z<y<3 z / 2$, we make the change of variable $t=y+z-2 s$ and write

$$
\begin{aligned}
S_{\alpha}(y, z)= & A(y z)^{1-2 v} \int_{0}^{z / 2}[(y+z-s)(y-s)(z-s) s]^{\nu-1}(y+z-2 s)^{-2 \alpha-2 v} d s \\
& +A(y z)^{1-2 v} \int_{z / 2}^{2 z-y}[(y+z-s)(y-s)(z-s) s]^{\nu-1}(y+z-2 s)^{-2 \alpha-2 v} d s \\
& +A(y z)^{1-2 v} \int_{2 z-y}^{z}[(y+z-s)(y-s)(z-s) s]^{\nu-1}(y+z-2 s)^{-2 \alpha-2 v} d s \\
& =A\left[I_{1}+I_{2}+I_{3}\right] .
\end{aligned}
$$


Let us write $w \cong v$ if $w / v$ is bounded between positive constants. Considering the integrand in $I_{1}$, where $0 \leqq s \leqq z / 2$, we have

$$
\begin{aligned}
(y+z-2 s) & \cong y, \\
(z-s) & \cong y \quad \text { and }
\end{aligned}
$$

$$
\begin{aligned}
(y+z-s) & \cong y \\
(y-s) & \cong y .
\end{aligned}
$$

It follows that

$$
\begin{aligned}
I_{1} & \leqq A y^{-\nu-2 \alpha-2} z^{1-2 \nu} \int_{0}^{z / 2} s^{\nu-1} d s=A y^{-\nu-2 \alpha-2} z^{1-\nu} \\
& \leqq A(y-z)^{-1-2 \alpha} z^{-2 \nu} \leqq A(y-z)^{-1-2 \alpha}(y+z)^{-2 \nu}
\end{aligned}
$$

since $y-z \leqq y, y \cong z$, and $y>(1 / 2)(y+z)$.

Considering the integrand of $I_{2}$, where $z / 2 \leqq s \leqq 2 z-y$, we have
(a)
$(z-s) \cong(y-s)$,
$(y+z-2 s) \cong(y-s)$,
(c)
$(y+z-s) \cong y \quad$ and
$s \cong y$.

It follows that

$$
\begin{aligned}
I_{2} & \leqq A y^{-1} z^{1-2 \nu} \int_{z / 2}^{2 z-y}(y-s)^{-2-2 \alpha} d s=A y^{-1} z^{1-2 v}(y-z)^{-1-2 \alpha} \\
& \leqq A(y-z)^{-1-2 \alpha} z^{-2 \nu} \leqq A(y-z)^{-1-2 \alpha}(y+z)^{-2 \nu}
\end{aligned}
$$

Considering the integrand of $I_{3}$, where $2 z-y \leqq s \leqq z$, we see that
(a)
$(y-s) \cong(y-z)$,
(b)
$(y+z-2 s) \cong(y-z)$,
(c)
$(y+z-s) \cong y, \quad$ and
$s \cong y$.

Therefore we obtain

$$
\begin{aligned}
I_{3} & \leqq A y^{-1} z^{1-2 \nu}(y-z)^{-1-2 \alpha-\nu} \int_{2 z-\nu}^{z}(z-s)^{\nu-1} d s \\
& \leqq A(y-z)^{-1-2 \alpha} z^{-2 v} \leqq A(y-z)^{-1-2 \alpha}(y+z)^{-2 \nu} .
\end{aligned}
$$

Combining these results, we have the inequality under the assumption that $z<y<3 z / 2$.

We next suppose that $y \geqq 3 z / 2$. We then obtain

$$
\begin{aligned}
& \text { (a) }(y+z-2 s) \cong y, \\
& \text { (b) }(y+z-s) \cong y, \text { and } \\
& \text { (c) } \quad(y-s) \cong y .
\end{aligned}
$$

Using these relations together with the inequality $y \geqq 3(y+z) / 5$, we conclude that 


$$
\begin{aligned}
I_{3} & \leqq A y^{-1-2 \alpha-2 \nu} z^{1-2 \nu} \int_{0}^{z}(z-s)^{\nu-1} s^{\nu-1} \\
& \leqq A(y-z)^{-1-2 \alpha} y^{-2 \nu} \int_{0}^{1}(1-t)^{\nu-1} t^{\nu-1} d t \\
& \leqq A(y-z)^{-1-2 \alpha}(y+z)^{-2 \nu} .
\end{aligned}
$$

LeMma 3B. If $f \in H_{\nu}^{\alpha, 2}, 0<\alpha<1 / 2, \nu>0$, and

$$
S_{a, b}[f(x)]=\int_{a}^{b} f^{\wedge}(y) V_{\nu}(x y) d m_{\nu}(y), \quad 0 \leqq a<b<\infty,
$$

then

$$
\left\|S_{a, b}[f]\right\|_{\alpha, 2} \leqq A(\alpha, \nu)\|f\|_{\alpha, 2}
$$

Proof. Let $J=[a, b]$ and $J^{\prime}$ be the complement of $J$ in $[0, \infty)$. By Theorem $2 \mathrm{C}$ we have

$$
\begin{aligned}
\left\|S_{a, b}[f]\right\|_{\alpha, 2}^{2}= & \frac{1}{2} \int_{J} \int_{J}\left[f^{\wedge}(y)-f^{\wedge}(z)\right]^{2} S_{\alpha}(y, z) d m_{\nu}(y) d m_{\nu}(z) \\
& +\frac{1}{2} \int_{J^{\prime}} \int_{J} f^{\wedge}(y)^{2} S_{\alpha}(y, z) d m_{\nu}(y) d m_{\nu}(z) \\
& +\frac{1}{2} \int_{J} \int_{J^{\prime}} f^{\wedge}(z)^{2} S_{\alpha}(y, z) d m_{\nu}(y) d m_{\nu}(z) \\
= & I_{1}+I_{2}+I_{3} .
\end{aligned}
$$

Using Theorem $2 \mathrm{C}$ again we obtain

$$
I_{1} \leqq \frac{1}{2} \int_{0}^{\infty} \int_{0}^{\infty}\left[f^{\wedge}(y)-f^{\wedge}(z)\right]^{2} S_{\alpha}(y, z) d m_{\nu}(y) d m_{\nu}(z)=\|f\|_{\alpha, 2}^{2} .
$$

By Lemma 3A and Corollary 1D we also obtain

$$
\begin{aligned}
I_{3} & \leqq A \int_{J} f^{\wedge}(z)^{2} d m_{\nu}(z) \int_{J^{\prime}}|y-z|^{-1-2 \alpha} d y \\
& =A \int_{J} f^{\wedge}(z)^{2}(b-z)^{-2 \alpha} d m_{\nu}(z)+A \int_{J} f^{\wedge}(z)^{2}(z-a)^{-2 \alpha} d m_{\nu}(z) \\
& \leqq A\|f\|_{\alpha, 2}^{2} .
\end{aligned}
$$

Using Fubini's theorem and symmetry $I_{2}=I_{3}$. This completes the proof.

For $n=0, \pm 1, \pm 2, \cdots$, let us set $b_{n}=3 \cdot 2^{n-2}, r_{n}=2^{n-1}, \sigma_{n}=\left[b_{n}-r_{n}, b_{n}+r_{n}\right]$, $\rho_{n}(y)=1-\left(y-b_{n}\right)^{2} r_{n}^{-2}$, and 


$$
E_{n}(x)=\int_{o_{n}} \rho_{n}(y) f^{\wedge}(y) V_{\nu}(x y) d m_{\nu}(y) .
$$

Lemma 3C. If $f \in H_{\nu}^{\alpha, 2}, 0<\alpha<1 / 2, \nu>0$, then

$$
\sum_{-\infty}^{\infty}\left\|E_{n}\right\|_{\alpha, 2}^{2} \leqq A(\alpha, \nu)\|f\|_{\alpha, 2}^{2} .
$$

Proof. By Theorem 2C we have

$$
\begin{aligned}
\left\|E_{n}\right\|_{\alpha, 2}^{2}= & \frac{1}{2} \int_{\sigma_{n}} \int_{\sigma_{n}}\left[\rho_{n}(y) f^{\wedge}(y)-\rho_{n}(z) f^{\wedge}(z)\right]^{2} S_{\alpha}(y, z) d m_{\nu}(y) d m_{\nu}(z) \\
& +\frac{1}{2} \int_{\sigma_{n}^{\prime}} \int_{\sigma_{n}} \rho_{n}(y)^{2} f^{\wedge}(y)^{2} S_{\alpha}(y, z) d m_{\nu}(y) d m_{\nu}(z) \\
& +\frac{1}{2} \int_{\sigma_{n}} \int_{\sigma^{\prime} n} \rho_{n}(z)^{2} f^{\wedge}(z)^{2} S_{\alpha}(y, z) d m_{\nu}(y) d m_{\nu}(z) \\
= & I_{1, n}+I_{2, n}+I_{3, n} .
\end{aligned}
$$

By Lemma 3A we obtain

$$
\begin{aligned}
\int_{\sigma_{n}^{\prime}} S_{\alpha}(y, z) d m_{\nu}(y) & \leqq A \int_{\sigma_{n}^{\prime}}|y-z|^{-1-2 \alpha} d y \\
& =A\left(z-b_{n}+r_{n}\right)^{-2 \alpha}+A\left(r_{n}+b_{n}-z\right)^{-2 \alpha} .
\end{aligned}
$$

For any $n$ and any $z \in \sigma_{n}$ the following are easy to establish:

$$
\begin{array}{cl}
\sigma_{n}(z)^{2} \leqq 4\left(r_{n}-b_{n}+z\right)^{2} r_{n}^{-2}, & \sigma_{n}(z)^{2} \leqq 4\left(r_{n}+b_{n}-z\right)^{2} r_{n}^{-2} \\
\left(r_{n}+b_{n}-z\right)^{2-2 \alpha} \leqq A z^{2-2 \alpha}, & \left(r_{n}-b_{n}+z\right)^{2-2 \alpha} \leqq A z^{2-2 \alpha}
\end{array}
$$

and

$$
r_{n}^{-2} \leqq A z^{-2} .
$$

Therefore we have

$$
\int_{\sigma^{\prime}{ }^{\prime}} \rho_{n}(z)^{2} S_{\alpha}(y, z) d m_{\nu}(y) \leqq A z^{-2 \alpha} .
$$

Noting that no point is in more than three of the sets $\sigma_{n}$ and using Theorem $1 \mathrm{~B}$, we obtain

$$
\begin{aligned}
\sum_{-\infty}^{\infty} I_{3, n} & \leqq A \sum_{-\infty}^{\infty} \int_{\sigma_{n}} f^{\wedge}(z)^{2} z^{-2 \alpha} d m_{\nu}(z) \\
& \leqq A \int_{0}^{\infty} f^{\wedge}(z)^{2} z^{-2 \alpha} d m_{\nu}(z) \leqq A\|f\|_{\alpha, 2 .}^{2} .
\end{aligned}
$$


A similar inequality may be obtained for the sum of $I_{2, n}$.

We next consider $I_{1, n}$. Since $\rho_{n}(y) \leqq 1$ when $y \in \sigma_{n}$, we see that

$$
\begin{aligned}
I_{1, n} \leqq & \int_{\sigma_{n}} \int_{\sigma_{n}}\left[f^{\wedge}(y)-f^{\wedge}(z)\right]^{2} S_{\alpha}(y, z) d m_{\nu}(y) d m_{\nu}(z) \\
& +\int_{\sigma_{n}} \int_{\sigma_{n}} f^{\wedge}(z)^{2}\left[\rho_{n}(y)-\rho_{n}(z)\right]^{2} S_{\alpha}(y, z) d m_{\nu}(y) d m_{\nu}(z) \\
= & I_{1, n}^{\prime}+I_{1, n}^{\prime \prime} .
\end{aligned}
$$

Using Theorem $2 \mathrm{C}$, we have

$$
\begin{aligned}
\sum_{-\infty}^{\infty} I_{1, n}^{\prime} & \leqq \int_{0}^{\infty} \int_{0}^{\infty}\left[f^{\wedge}(y)-f^{\wedge}(z)\right]^{2} S_{\alpha}(y, z) d m_{\nu}(y) d m_{\nu}(z) \\
& =2\|f\|_{\alpha, 2}^{2} .
\end{aligned}
$$

By the definition of $\sigma_{n}$, we see that for $y$ and $z$ in $\sigma_{n}$

$$
\begin{aligned}
{\left[\rho_{n}(y)-\rho_{n}(z)\right]^{2} } & =(y-z)^{2}\left(y+z-2 b_{n}\right)^{2} r_{n}^{-4} \\
& \leqq A(y-z)^{2} r_{n}^{-2} .
\end{aligned}
$$

Using the inequalities (3) we obtain for the inner integral of $I_{1, n}^{\prime \prime}$

$$
\begin{aligned}
& \int_{\sigma_{n}}\left[\rho_{n}(y)-\rho_{n}(z)\right]^{2} S_{\alpha}(y, z) d m_{\nu}(y) \\
& \quad \leqq A r_{n}^{-2} \int_{\sigma_{n}}|y-z|^{1-2 \alpha} d y \\
& \quad \leqq A r_{n}^{-2}\left(b_{n}+r_{n}-z\right)^{2-2 \alpha}+A r_{n}^{-2}\left(r_{n}-b_{n}+z\right)^{2-2 \alpha} \\
& \quad \leqq A z^{-2 \alpha} .
\end{aligned}
$$

We may now conclude by Theorem $1 B$ that

$$
\sum_{-\infty}^{+\infty} I_{1, n}^{\prime \prime} \leqq A \sum_{-\infty}^{+\infty} \int_{0_{n}} f^{\wedge}(z)^{2} z^{-2 \alpha} d m_{\nu}(z) \leqq A\|f\|_{\alpha, 2}^{2} .
$$

LEMmA 3D. If $2^{n-1} \leqq z_{n} \leqq 2^{n},-\infty<n<\infty$, and $f(x) \in H_{v}^{\alpha, 2}, 0<\alpha<1 / 2$, $\nu>0$, then

$$
\sum_{-\infty}^{+\infty} \int_{2^{n-1}}^{2^{n}} f^{\wedge}(z)^{2}\left|z_{n}-z\right|^{-2 \alpha} d m_{\nu}(z) \leqq A(\alpha, \nu)\|f\|_{\alpha, 2}^{2} .
$$

Proof. We note that if $2^{n-1} \leqq z \leqq 2^{n}$, then $\rho_{n}(z) \geqq 3 / 4$. Thus we have 


$$
\begin{aligned}
\int_{2^{n-1}}^{2^{n}} f^{\wedge}(z)^{2}\left|z_{n}-z\right|^{-2 \alpha} d m_{\nu}(z) & \leqq A \int_{2^{n-1}}^{2^{n}} \rho_{n}(z)^{2}\left|z_{n}-z\right|^{-2 \alpha} d m_{\nu}(z) \\
& =A \int_{0}^{\infty} E_{n}^{\wedge}(z)^{2}\left|z_{n}-z\right|^{-2 \alpha} d m_{\nu}(z) \\
& \leqq A\left\|E_{n}\right\|_{\alpha, 2}^{2}
\end{aligned}
$$

by Corollary 1D. Application of Lemma 3C completes the proof.

Theorem 3E. If $f(x) \in H_{\nu}^{\alpha, 2}(0, \infty),-1 / 2<\alpha<1 / 2, \nu>0$, and if $\phi(y)$ has the properties

$$
|\phi(y)| \leqq C, \quad 0 \leqq y \leqq \infty,
$$

and

$$
\int_{2^{n-1}}^{2^{n}}|d \phi(y)| \leqq C, \quad-\infty<n<\infty
$$

then the Hankel multiplier transformation $T$ associated with $\phi(y)$ exists on $H_{\nu}^{\alpha, 2}(0, \infty)$ and

$$
\|T f\|_{\alpha, 2} \leqq A(\alpha, \nu) C\|\|_{\alpha, 2} .
$$

Proof. The result is trivial for $\alpha=0$. Next suppose that $0<\alpha<1 / 2$ and that $f \in H_{\nu}^{\alpha, 2} \cap H_{\nu}^{2}$. Let $\Delta_{n}(x)$ be the Hankel multiplier transform of $f$ associated with a function equal to $\phi(y)$ on $\left[2^{n-1}, 2^{n}\right]$ and zero elsewhere for $-\infty<n<\infty$. Also let $F_{N}(x)$ be the sum of $\Delta_{n}(x)$ from $-N$ to $N, N$ $=1,2, \cdots$. We first show that independently of $N$

$$
\left\|F_{N}\right\|_{\alpha, 2} \leqq A C\|f\|_{\alpha, 2} \text {. }
$$

To simplify writing we omit the subscripts $\alpha, 2$, and $\nu$. Since

$$
\left\|F_{N}\right\|^{2}=\sum_{-N}^{+N} \int_{0}^{\infty} \Delta_{n}(x)^{2} x^{2 \alpha} d m(x)+\sum_{n, m=-N ; n \neq m}^{+N} \int_{0}^{\infty} \Delta_{n}(x) \Delta_{m}(x) x^{2 \alpha} d m(x),
$$

the inequality (a) is implied by the inequalities

$$
\sum_{-N}^{+N}\left\|\Delta_{n}\right\|^{2} \leqq A C^{2}\|f\|^{2}
$$

and

$$
\sum_{n, m=-N ; n \neq m}^{N}\left|\int_{0}^{\infty} \Delta_{n}(x) \Delta_{m}(x) x^{2 \alpha} d m(x)\right| \leqq A C^{2}\|f\|^{2} .
$$

To prove (b) we suppose $z \in \sigma_{n}$ and set 


$$
S_{z}^{(n)}(x)=S_{2^{-N-1, z}}\left[E_{n}(x)\right]=\int_{b_{n}-r_{n}}^{z} \rho_{n}(y) f^{\wedge}(y) V(x y) d m(y) .
$$

By Lemma 3B we have

$$
\left\|S_{z}^{(n)}\right\| \leqq A\left\|E_{n}\right\| .
$$

Setting $\phi_{n}(z)=\phi(z) / \rho_{n}(z)$ and integrating by parts we obtain

$$
\begin{aligned}
\Delta_{n}(x) & =\int_{2^{n-1}}^{2^{n}} \phi(z) f^{\wedge}(z) V(x z) d m(z)=\int_{2^{n-1}}^{2^{n}} \phi_{n}(z) d_{z} S_{z}^{(n)}(x) \\
& =S_{2^{n}}^{(n)}(x) \phi_{n}\left(2^{n}\right)-S_{2^{n-1}}^{(n)}(x) \phi_{n}\left(2^{n-1}\right)-\int_{2^{n-1}}^{2 n} S_{z}^{(n)}(x) d \phi_{n}(z) .
\end{aligned}
$$

Since for all $n,\left|\phi_{n}(z)\right| \leqq A C$ and $\int_{2^{n-1}}^{2^{n}}\left|d \phi_{n}(z)\right| \leqq A C$, we see that by Minkowski's inequalities for sums and for integrals, Hardy, Littlewood, and Polya [1952, p. 148], and by Lemma 3B

$$
\begin{aligned}
\left\|\Delta_{n}(x)\right\| & \leqq C\left\|S_{2^{n}}^{(n)}(x)\right\|+C\left\|S_{2^{n-1}(x)}^{(n)}\right\|+\int_{2^{n-1}}^{2^{n}}\left\|S_{z}^{(n)}(x)\right\|\left|d \phi_{n}(z)\right| \\
& \leqq A C\left\|E_{n}(x)\right\| .
\end{aligned}
$$

Using Lemma $3 \mathrm{C}$, we now obtain

$$
\sum_{-N}^{N}\left\|\Delta_{n}\right\|^{2} \leqq A C^{2} \sum_{-N}^{N}\left\|E_{n}\right\| \leqq A C^{2}\|f\|
$$

proving (b).

We next consider (c). Let

$$
I_{m, n}=\int_{0}^{\infty} \Delta_{n}(x) \Delta_{m}(x) x^{2 \alpha} d m(x)
$$

for each pair of distinct integers. Setting $J_{n}=\left[2^{n-1}, 2^{n}\right]$, we have by Corollary $2 \mathrm{D}$

$$
\begin{aligned}
I_{m, n}= & -\frac{1}{2} \int_{J_{m}} \int_{J_{n}} \phi(y) f^{\wedge}(y) \phi(z) f^{\wedge}(z) S(y, z) d m(y) d m(z) \\
& -\frac{1}{2} \int_{J_{n}} \int_{J_{m}} \phi(y) f^{\wedge}(y) \phi(z) f^{\wedge}(z) S(y, z) d m(y) d m(z) \\
= & I_{m, n}^{\prime}+I_{m, n}^{\prime \prime} .
\end{aligned}
$$

We have also used the fact that $J_{n} \cap J_{m}$ is of measure zero. This equality will in fact be valid only after we have shown that the integrals involved are finite. 
Using the estimate $|a b| \leqq(1 / 2) a^{2}+(1 / 2) b^{2}$, we obtain

$$
\begin{aligned}
\left|I_{m, n}^{\prime}\right| \leqq & A C^{2} \int_{J_{m}} \int_{J_{n}} f^{\wedge}(y)^{2} S(y, z) d m(y) d m(z) \\
& +A C^{2} \int_{J_{m}} \int_{J_{n}} f^{\wedge}(z)^{2} S(y, z) d m(y) d m(z) \\
= & I_{m, n}^{*}+I_{m, n}^{* *} .
\end{aligned}
$$

By Lemmas 3A and 3D we see that

$$
\begin{aligned}
\sum_{n, m=-N i n \neq m}^{N}\left|I_{m, n}^{* *}\right| \leqq & A C^{2} \sum_{m=-N}^{N} \int_{J_{m}} f^{\wedge}(z)^{2}\left[\sum_{n=-N ; n \neq m}^{N} \int_{J_{n}} S(y, z) d m(y)\right] d m(z) \\
\leqq & A C^{2} \sum_{m=-N}^{N} \int_{J_{m}} f^{\wedge}(z)^{2}\left(z-2^{m-1}\right)^{-2 \alpha} d m(z) \\
& +A C^{2} \sum_{m=-N}^{N} \int_{J_{m}} f^{\wedge}(z)^{2}\left(2^{m}-z\right)^{-2 \alpha} d m(z) \\
\leqq & A C^{2}\|f\| .
\end{aligned}
$$

Similarly

$$
\sum_{n, m-N ; n \ngtr m}^{N}\left|I_{m, n}^{*}\right| \leqq A C^{2}\|f\|
$$

and therefore we have

$$
\sum_{n, m=-N}^{N}\left|I_{m, n}^{\prime}\right| \leqq A C^{2}\|f\|
$$

By symmetry we also obtain

$$
\sum_{n, m=-N}^{N}\left|I_{m, n}^{\prime \prime}\right| \leqq A C^{2}\|f\| .
$$

The inequality (c) now follows from (6), (7), and (8). Thus we also have (a). Let $T f$ denote the limit in $H_{\nu}^{\alpha, 2}$ of $F_{N}$ as $N \rightarrow \infty$. We then have

$$
\|T f\| \leqq A C\|f\|
$$

and

$$
[T f]^{\wedge}(y)=\phi(y) f^{\wedge}(y)
$$

for every $f \in H_{\nu}^{\alpha, 2} \cap H_{\nu}^{2}, 0<\alpha<1 / 2$.

Next suppose that $-1 / 2<\alpha<0, f \in H_{\nu}^{\alpha, 2} \cap H_{\nu}^{2}$, and $g \in H_{\nu}^{-\alpha, 2} \cap H_{\nu}^{2}$. Using Plancherel's theorem, the Schwarz inequality, and what we have just proved, we have 


$$
\begin{aligned}
\int_{0}^{\infty}[T f(x)] g(x) d m(x) & =\int_{0}^{\infty} \phi(y) f^{\wedge}(y) g^{\wedge}(y) d m(y) \\
& =\int_{0}^{\infty} f(x)[T g(x)] d m(x) \\
& \leqq\|f\|_{\alpha, 2}\|T g\|_{-\alpha, 2} \leqq A C\|f\|_{\alpha, 2}\|g\|_{-\alpha, 2} .
\end{aligned}
$$

Supposing in addition that $\|g\|_{-\alpha, 2} \leqq 1$, we obtain by a well known formula

$$
\|T f\|_{\alpha, 2}=\underset{\sigma}{\text { l.u.b. }} \int_{0}^{\infty}[T f(x)] g(x) d m(x) \leqq A C\|f\|_{\alpha, 2} .
$$

We now have (9) and (10) for all $f$ in $H_{\nu}^{\alpha, 2} \cap H_{\nu}^{2}$. Since this is a dense subspace of $H_{\nu}^{\alpha, 2}, T$ has a unique extension to $H_{\nu}^{\alpha, 2}$ such that (9) holds.

\section{Part II. Weighted $p$-Norms}

In this part we extend the main result, Theorem $3 \mathrm{E}$, of Part I to the case of weighted $p$-norms. We let $L^{\alpha, p}(a, b)$ denote the space of all complex valued functions $f$ measurable on $(a, b)$ and such that the weighted $p$-norm

$$
\|f\|_{\alpha, p}=\left[\int_{a}^{b}|f(x)|^{p}|x|^{\alpha p} d x\right]^{1 / p}<\infty .
$$

We show in Theorem $8 \mathrm{D}$, that if $f \in L^{\alpha, p}(0, \infty)$ and if $\phi$ satisfies the conditions given in Theorem $3 \mathrm{E}$, then the ordinary Hankel multiplier transformation $T_{\nu}$ of order $\nu \geqq-1 / 2$ is defined on $L^{\alpha, p}$ and $\left\|T_{\nu} f\right\|_{\alpha, p} \leqq A(\alpha, p, \nu)\|f\|_{\alpha, p}$ for all $p>1$ and $-1 / p<\alpha<1-1 / p$.

The methods of Part I, depending on the condition $p=2$ are not available to us here. Instead we first prove the corresponding result for Fourier multiplier transformations on $L^{\alpha, p}(-\infty, \infty)$. The methods used are essentially those of the proof of the corresponding result for Fourier series multiplier transformations on $L^{\alpha, p}(-\pi, \pi)$, Marcinkiewicz [1939, p. 78], Hirschman [1955a, p. 29]. An essential step in the proof is a decomposition of the Fourier integral, derived in $\S 7$. The last section contains the main theorems on Fourier and Hankel multiplier transformations. $\S \S 4,5$, and 6 are devoted to preliminaries.

4. Conjugates and partial integrals. We begin with a consideration of a famous result of M. Riesz and derive several consequences from it. Suppose that $f \in L^{1} \cap L^{2} \cap L^{\alpha, p}$ under the usual restrictions on $\alpha$ and $p$. Consider the transformation which sends $f$ into

$$
f^{\sim}(x)=(2 \pi)^{-1 / 2} \int_{-\infty}^{\infty} i \operatorname{sgn} t f^{\wedge}(t) e^{-i x t} d t,
$$

where $f^{\wedge}$ is the Fourier transform of $f$. Since $i \operatorname{sgn} t$ is bounded and $f \in L^{2}$, $f^{\sim}$ is clearly defined, at least as a limit in $L^{2}$ of partial integrals. 
Moreover it is well known, Titchmarsh [1957, p. 120], that this expression may be written in the form

$$
f^{\sim}(x)=\pi^{-1} \int_{-\infty}^{\infty} f(u)(x-u)^{-1} d u
$$

the integral being the Cauchy mean value at $u=x$. This function is known as the conjugate of $f$; its negative is called the Hilbert transform, Tirchmarsh [1937, p. 120].

We shall make use of the following generalization, due to Hardy and Littlewood [1936, p. 370], of a result first proved in the case $\alpha=0$ by $M$. Riesz $[1923 ; 1927]$ :

Theorem 4A. (Hardy, Littlewood, Riesz). If $f \in L^{\alpha, p}(-\infty, \infty)$, $1<p<\infty,-1 / p<\alpha<1-1 / p$, then $\tilde{f}^{\sim}$ as given by (2) exists and

$$
\left\|f^{\sim}\right\|_{\alpha, p} \leqq A(\alpha, p)\|f\|_{\alpha, p} .
$$

We next define

$$
S_{a, b}[f(x)]=(2 \pi)^{-1 / 2} \int_{a}^{b} f^{\wedge}(t) e^{-i x t} d t
$$

for $-\infty<a<b<\infty$ and $f \in L^{1} \cap L^{\alpha, p}$ and prove

Lemma 4B. If $f \in L^{\alpha, p}(-\infty, \infty), 1<p<\infty,-1 / p<\alpha<1-1 / p$, then $S_{a, b}$ has an extension to $L^{\alpha, p}$ so that

$$
\left\|S_{a, b}[f]\right\|_{\alpha, p} \leqq A(\alpha, p)\|f\|_{\alpha, p} .
$$

Proof. Suppose at first that $f \in L^{1} \cap L^{\alpha, p}$. Using Fubini's theorem and (2), we obtain

$$
\begin{aligned}
S_{a, b}[f(x)]= & (2 \pi)^{-1} \int_{a}^{b} e^{-i x t} d t \int_{-\infty}^{\infty} f(u) e^{i t u} d u \\
= & (2 \pi)^{-1} \int_{-\infty}^{\infty} f(u) d u \int_{a}^{b} e^{i t(u-x)} d t \\
= & (2 \pi)^{-1} \int_{-\infty}^{\infty} f(u)\left[e^{i b(u-x)}-e^{i a(u-x)}\right](u-x)^{-1} d u \\
= & (2 \pi i)^{-1} e^{-i b x} \int_{-\infty}^{\infty} e^{i b u} f(u)(u-x)^{-1} d u \\
& -(2 \pi i)^{-1} e^{-i a x} \int_{-\infty}^{\infty} e^{i a u} f(u)(u-x)^{-1} d u \\
= & (i / 2)\left[e^{-i b x}\left(e^{i b x} f(x)\right)^{\sim}-\left(e^{-i a x} f(x)\right)^{\sim}\right] .
\end{aligned}
$$


By Minkowski's inequality and Theorem 4A we have

$$
\begin{aligned}
\left\|S_{a, b}[f]\right\| & \leqq 2^{-1}\left\|\left(e^{i b x} f(x)\right)^{\sim}\right\|+2^{-1}\left\|\left(e^{i a x} f(x)\right) \sim\right\| \\
& \leqq A(\alpha, p)\|f\| .
\end{aligned}
$$

Since $L^{1} \cap L^{\alpha, p}$ is dense in $L^{\alpha, p}$, the bounded linear transformation $S_{a, b}$ has a unique bounded linear extension to $L^{\alpha, p}$.

Lemмa 4C. Under the hypotheses of Lemma 4B,

$$
\underset{a \rightarrow \infty}{\lim .{ }^{\alpha, p} S_{a, b}[f]=f .}
$$

In the case $\alpha=0,1<p \leqq 2$, the proof is well known, Titchmarsh [1937, p. 149]. With trivial modification the same proof works for the present lemma as well.

The next result is a simplified version of the main theorem on Fourier multiplier transformations. See Stečkin [1950, p. 240].

TheoRem 4D. If $f \in L^{\alpha, p}(-\infty, \infty), 1<p<\infty,-1 / p<\alpha<1-1 / p$, and if $\phi$ has the properties

$$
|\phi(t)| \leqq C, \quad-\infty<t<\infty,
$$

and

$$
\int_{-\infty}^{\infty}|d \phi(t)| \leqq C
$$

then the Fourier multiplier transformation $T$ associated with $\phi$ is defined on $L^{\alpha, p}$ and

$$
\|T f\|_{\alpha, p} \leqq A(\alpha, p) C\|f\|_{\alpha, p} .
$$

Proof. Suppose that $f \in L^{2} \cap L^{\alpha, p}$ and set

$$
F_{a}(x)=(2 \pi)^{-1 / 2} \int_{-a}^{a} \phi(t) f^{\wedge}(t) e^{-i x t} d t .
$$

Using (3) and integration by parts, we obtain

$$
\begin{aligned}
F_{a}(x) & =\int_{-a}^{a} \phi(t) d S_{-a, t}[f(x)] \\
& =\phi(a) S_{-a, a}[f(x)]-\int_{-a}^{a} S_{-a, t}[f(x)] d \phi(t) .
\end{aligned}
$$

By Minkowski's inequalities for sums and for integrals we have, with the aid of Lemma 4B, 


$$
\begin{aligned}
\left\|F_{a}\right\| & \leqq\left\|\phi(a) S_{-a, a}[f]\right\|+\left\|\int_{-a}^{a} S_{-a, t}[f] d \phi(t)\right\| \\
& \leqq|\phi(a)|\left\|S_{-a, a}[f]\right\|+\int_{-a}^{a}\left\|S_{-a, t}[f]\right\||d \phi(t)| \\
& \leqq A C\|f\| .
\end{aligned}
$$

By similar arguments we have for $0<a^{\prime}<a$

$$
\begin{aligned}
\left\|F_{a}-F_{a^{\prime}}\right\| \leqq & C\left\|S_{-a, a}[f]-S_{-a^{\prime}, a^{\prime}}[f]\right\| \\
& +A\left|\phi(a)-\phi\left(a^{\prime}\right)\right|\|f\|+A \int_{a^{\prime} \leq|t| \leq a}|d \phi(t)|\|f\| .
\end{aligned}
$$

Using (6), (7), and Lemma $4 \mathrm{C}$, we see that $F_{a}$ is Cauchy in $L^{\alpha, p}$. Letting $T f$ denote its limit in $L^{\alpha, p}$ as $a \rightarrow \infty$, we obtain

$$
\|T f\| \leqq A C\|f\|
$$

for all $f \in L^{2} \cap L^{\alpha, p}$. That $T$ is a Fourier multiplier transformation is evident. The extension to $L^{\alpha, p}$ is standard.

Before proving our next theorem we establish some lemmas. These are all analogous to certain results of A. Zygmund [1938, p. 182] for Fourier series. We first quote a lemma due to Zygmund [1938, p. 176].

Lemma $4 \mathrm{E}$ (Zygmund). If $r_{n}(t)$ is the nth Rademacher function and $h(t)$ $=\sum_{1}^{N} a_{n} r_{n}(t)$ and if $1<p<\infty$, then $\left(^{5}\right)$

$$
\int_{0}^{1}|h(t)|^{p} d t \cong\left(\sum_{1}^{N}\left|a_{n}\right|^{2}\right)^{p / 2}
$$

Lemma $4 \mathrm{~F}$. If $f_{n} \in L^{\alpha, p}(-\infty, \infty), n=1, \cdots, N, 1<p<\infty,-1 / p<\alpha<1$ $-1 / p$, then $\left(\sum_{1}^{N}\left|f_{n}\right|^{2}\right)^{1 / 2} \in L^{\alpha, p}$ and

$$
\left\|\left(\sum_{1}^{N}\left|\tilde{f_{n}}\right|^{2}\right)^{1 / 2}\right\|_{\alpha, p} \leqq A(\alpha, p)\left\|\left(\sum_{1}^{N}\left|f_{n}\right|^{2}\right)^{1 / 2}\right\|_{\alpha, p} .
$$

The proof is the same as that of the corresponding result for Fourier series on $L^{p}(-\pi, \pi)$ in Zygmund [1938, p. 176].

Lemma 4G. Under the hypotheses of Lemma 4F,

$$
\left\|\left(\sum_{1}^{N}\left|S_{0, t_{n}}\left[f_{n}\right]\right|^{2}\right)^{1 / 2}\right\|_{\alpha, p} \leqq A(\alpha, p)\left\|\left(\sum_{1}^{N}\left|f_{n}\right|^{2}\right)^{1 / 2}\right\|_{\alpha, p}
$$

for any sequence $t_{1}, \cdots, t_{n}$ of positive reals.

Proof. By equation (5) we have

(5) By this notation we mean that the quotient of the expressions involved is bounded between positive constants. 


$$
S_{0, t_{n}}[f(x)]=\frac{i}{2}\left[e^{-i t_{n} x}\left(e^{i t_{n} x} f_{n}(x)\right)^{\sim}-f^{\sim}(x)\right]
$$

The result is immediate using the inequality $(a+b)^{2} \leqq 2 a^{2}+2 b^{2}$, Minkowski's inequality and Lemma $4 \mathrm{~F}$.

Theorem 4H. If $1<p<\infty,-1 / p<\alpha<1-1 / p, f_{n} \in L^{\alpha, p}(-\infty, \infty)$ and $\alpha_{n}$ is nondecreasing on an interval $\left[a_{n}, b_{n}\right]$ for $n=1, \cdots, N$, then

$$
\left\|\left(\sum_{1}^{N} \int_{a_{n}}^{b_{n}}\left|S_{0, t}\left[f_{n}\right]\right|^{2} d \alpha_{n}(t)\right)^{1 / 2}\right\|_{\alpha, p} \leqq A(\alpha, p)\left\|\left(\sum_{1}^{N}\left|f_{n}\right|^{2} \int_{a_{n}}^{b_{n}} d \alpha_{n}(t)\right)^{1 / 2}\right\|_{\alpha, p} .
$$

Proof. There is no loss of generality in supposing that $f_{n} \in L^{1} \cap L^{\alpha, p}$. Let each interval $\left[a_{n}, b_{n}\right]$ be partitioned into $m$ subintervals of length $\left(b_{n}-a_{n}\right) / m$ by points $t_{n, 1}, \cdots, t_{n, m-1}$. Let $t_{n, m}=b_{n}$ and set

$$
\Delta_{n, j}=\alpha_{n}\left(t_{n, j}\right)-\alpha_{n}\left(t_{n, j-1}\right)
$$

for $j=1,2, \cdots, m$. We then have

$$
\lim _{m \rightarrow \infty} \sum_{j=1}^{m}\left|S_{0, t_{n, j}}\left[f_{n}\right]\right|^{2} \Delta_{n, j}=\int_{a_{n}}^{b_{n}}\left|S_{0, t}\left[f_{n}\right]\right|^{2} d \alpha_{n}(t) .
$$

By Lemma $4 \mathrm{G}$ we see that

$$
\left\|\left(\sum_{n=1}^{N} \sum_{j=1}^{m} \mid S_{0, t_{n, j}}\left[f_{n}\right]^{2} \Delta_{n, j}\right)^{1 / 2}\right\|_{\alpha, p} \leqq A(\alpha, p)\left\|\left(\sum_{n=1}^{N}\left|f_{n}\right|^{2} \int_{a_{n}}^{b_{n}} d \alpha_{n}(t)\right)^{1 / 2}\right\|_{\alpha, p} .
$$

Using Fatou's lemma we obtain in view of (10)

$$
\begin{aligned}
& \left\|\left(\sum_{n=1}^{N} \int_{a_{n}}^{b_{n}}\left|S_{0, t}\left[f_{n}\right]\right|^{2} d \alpha_{n}(t)\right)^{1 / 2}\right\|_{\alpha, p} \\
& \leqq \liminf _{m \rightarrow \infty}\left\|\left(\sum_{n=1}^{N} \sum_{j=1}^{m}\left|S_{0, t_{n}, j}\left[f_{n}\right]\right|^{2} \Delta_{n, j}\right)^{1 / 2}\right\|_{\alpha, p} \\
& \leqq A(\alpha, p)\left\|\left(\sum_{n=1}^{N}\left|f_{n}\right|^{2} \int_{a_{n}}^{b_{n}} d \alpha_{n}(t)\right)^{1 / 2}\right\|_{\alpha, p} .
\end{aligned}
$$

5. The function $f(x, y)$. Suppose that $y>0$. If we set $\phi(t)=e^{-y|t|}$ in Theorem $4 \mathrm{D}$, then the associated multiplier transformation $T$ is defined on $L^{\alpha, p}$. If $f \in L^{\alpha, p}$, we let

$$
f(x, y)=T[f(x)]
$$

This section is devoted to the study of this function. then

Lemma 5A. If $f \in L^{\alpha, p}(-\infty, \infty), 1<p<\infty,-1 / p<\alpha<1-1 / p$, and $y>0$ 


$$
f(x, y)=\frac{1}{\pi} \int_{-\infty}^{\infty} f(u) y\left[(u-x)^{2}+y^{2}\right]^{-1} d u
$$

and

$$
f^{\sim}(x, y)=\frac{1}{\pi} \int_{-\infty}^{\infty} f(u)(u-x)\left[(u-x)^{2}+y^{2}\right]^{-1} d u .
$$

These formulae are established by routine calculations. Note that the Poisson kernel and its harmonic conjugate appear in (2) and (3) respectively. We shall denote these by $P(u, y)$ and $P^{\sim}(u, y)$. If we set $z=x+i y$ and

$$
F(z)=f(x, y)+i f^{\sim}(x, y),
$$

then it is easy to prove the following:

Corollary 5B. Under the hypotheses of Lemma 5A, $F(z)$ is analytic for $I(z)>0$.

Lemma 5C. If, under the hypotheses of Lemma 5A, we set

$$
f^{*}(x)=\underset{y>0}{\operatorname{l.u.b}}|f(x, y)|,
$$

then $\left\|f^{*}(x)\right\|_{\alpha, p} \leqq A(\alpha, p)\|f(x)\|_{\alpha, p}$.

Proof $\left({ }^{6}\right)$. We may suppose that $f(x) \geqq 0$ for all $x$. We set

$$
h(x)=\underset{-\infty<u<\infty}{\text { l.u.b. }} \frac{1}{u} \int_{0}^{u} f(x+t) d t .
$$

Integrating by parts and using the estimates

$$
\left|\int_{0}^{u} f(x+t) d t\right| \leqq\|f(x)\|_{\alpha, p} u^{1-1 / p-\alpha}
$$

and $P(u, y)=O\left(u^{-2}\right)$ as $|u| \rightarrow \infty$ we have

$$
\begin{aligned}
|f(x, y)| & =\left|\int_{-\infty}^{\infty} f(x+u) P(u, y) d u\right| \\
& =\left|\int_{-\infty}^{\infty} u P_{u}(u, y)\left[u^{-1} \int_{0}^{u} f(x+t) d t\right] d u\right| \\
& \leqq h(x) \int_{-\infty}^{\infty}\left|u P_{u}(u, y)\right| d u=A h(x) .
\end{aligned}
$$

It follows that $f^{*}(x) \leqq A h(x)$ and thus we see that

(6) This result is stated without proof in Waterman $[1956$, p. 170]. Since there appears to be no proof in the literature, we give one communicated to the author by I. I. Hirschman, Jr. 


$$
\left\|f^{*}\right\|_{\alpha, p} \leqq A\|h\|_{\alpha, p}
$$

We next show that

$$
\|h\|_{\alpha, p} \leqq A\left\|_{f}\right\|_{\alpha, p}
$$

Suppose that $x>0$ and set

$$
h_{1}(x)=\underset{u>0}{\text { l.u.b. }} \frac{1}{u} \int_{0}^{u} f(x+t) d t .
$$

Let $h_{2}(x)$ and $h_{3}(x)$ be least upper bounds of the same expression over $(-x, 0)$ and $(-\infty,-x)$ respectively.

Considering $h_{1}$ first, we set

$$
F_{1}(x)=\underset{u>2^{n}}{\text { l.u.b. }} \frac{1}{u} \int_{0}^{u} f(x+t) d t \text { and } G_{1}(x)=\underset{0<u<2^{n}}{\operatorname{lou}} \frac{1}{u} \int_{0}^{u} f(x+t) d t
$$

when $x \in\left[2^{n-1}, 2^{n}\right], n$ an integer. Setting $u=X-x$, we obtain

$$
G_{1}(x)=\underset{0<X-x<2^{n}}{\text { l.u.b. }} \frac{1}{X-x} \int_{0}^{X-x} f(x+t) d t \leqq \underset{\substack{\text { l.u.b. } \\ 0}}{\text { l.1 }<X<2^{n+1}} \frac{1}{X-x} \int_{x}^{x} f(v) d v .
$$

Using a theorem of Hardy and Littlewood, Zygmund [1935, p. 244], we have

$$
\begin{aligned}
\int_{2^{n-1}}^{2^{n}} G_{1}(x)^{p} d x & \leqq \int_{2^{n-1}}^{2^{n+1}}\left[\underset{2^{n-1}<X<2^{n+1}}{\text { l.u.b. }} \frac{1}{X-x} \int_{x}^{x} f(v) d v\right]^{p} d x \\
& \leqq A(p) \int_{2^{n-1}}^{2^{n+1}} f(x)^{p} d x .
\end{aligned}
$$

Since $x^{\alpha p} \cong 2^{\alpha n p}$ when $x \in\left[2^{n-1}, 2^{n+1}\right]$, we see that

$$
\int_{2^{n-1}}^{2^{n}} G_{1}(x)^{p} x^{\alpha p} d x \leqq A(\alpha, p) \int_{2^{n-1}}^{2^{n+1}} f(x)^{p} x^{\alpha p} d x
$$

Summing over all integers, we have

$$
\int_{0}^{\infty} G_{1}(x)^{p} x^{\alpha p} d x \leqq A(\alpha, p) \int_{0}^{\infty} f(x)^{p} x^{\alpha p} d x .
$$

In considering $F_{1}$ we set

$$
I_{n}=\int_{2^{n-1}}^{2^{n}} f(x) d x \quad \text { and } \quad c_{n}^{p}=\int_{2^{n-1}}^{2^{n}} f(x)^{p} x^{\alpha p} d x .
$$

Letting $q$ be the conjugate index of $p$ we see that by Hölder's inequality

$$
I_{n} \leqq\left[\int_{2^{n-1}}^{2^{n}} f(x)^{p} x^{\alpha p} d x\right]^{1 / p}\left[\int_{2^{n-1}}^{2^{n}} x^{-\alpha p} d x\right]^{1 / q} \leqq A c_{n} 2^{-\alpha n+n / q} .
$$


Since

$$
F_{1}(x) \leqq \underset{N \geqq n}{1 . u . b .} 2^{-N} \sum_{k=n}^{N+1} I_{k} \leqq 2 \sum_{k=n}^{\infty} 2^{-k} I_{k},
$$

we obtain using (8)

$$
F_{1}(x) \leqq A \sum_{k=m}^{\infty} 2^{-k-\alpha k+k / q} c_{k}=A \sum_{k=n}^{\infty} 2^{r k} c_{k}
$$

where $r=-1-\alpha+1 / q=-1 / p-\alpha<0$. It follows that

$$
\int_{2^{n-1}}^{2^{n}} F_{1}(x)^{p} x^{\alpha p} d x \leqq A \cdot 2^{\alpha p n+n}\left[\sum_{k=n}^{\infty} 2^{r k} c_{k}\right]^{p}=A\left[\sum_{k=n}^{\infty} 2^{r(k-n)} c_{k}\right]^{p} .
$$

Summing over all $n$, we see that

$$
\int_{0}^{\infty} F_{1}(x)^{p} x^{\alpha p} d x \leqq A \sum_{n=-\infty}^{\infty}\left[\sum_{k=n}^{\infty} 2^{r(k-n)} c_{k}\right]^{p}=A \sum_{n=-\infty}^{\infty}\left[b_{n} * c_{n}\right]^{p}
$$

where $b_{n}=2^{-r n}$ if $n \leqq 0$ and is zero otherwise. By an inequality of W. H. Young, (see Hardy, Littlewood, and Polya [1952, p. 198]), we obtain

$$
\sum_{n=-\infty}^{\infty}\left[b_{n} * c_{n}\right]^{p} \leqq\left[\sum_{n=-\infty}^{\infty} b_{n}\right]^{p} \sum_{n=-\infty}^{\infty} c_{n}^{p}=A(\alpha, p) \sum_{n=-\infty}^{\infty} c_{n}^{p}
$$

We now have

$$
\int_{0}^{\infty} F_{1}(x)^{p} x^{\alpha p} d x \leqq A(\alpha, p) \sum_{n=-\infty}^{\infty} c_{n}=A(\alpha, p) \int_{0}^{\infty} f(x)^{p} x^{\alpha p} d x .
$$

Combining (7) and (9) we see that

$$
\int_{0}^{\infty} h_{1}(x) x^{\alpha p} d x \leqq A(\alpha, p) \int_{0}^{\infty} f(x)^{p} x^{\alpha p} d x .
$$

By nearly the same arguments we may show that

$$
\int_{0}^{\infty} h_{2}(x) x^{\alpha p} d x \leqq A(\alpha, p) \int_{0}^{\infty} f(x) x^{\alpha p} d x .
$$

Finally we consider $h_{3}$ and obtain

$$
h_{3}(x) \leqq \frac{1}{x} \int_{0}^{x} f(u) d u+\frac{1}{x} \int_{0}^{x} f(-u) d u+\underset{u>0}{\text { l.u.b. }} \frac{1}{u} \int_{0}^{u} f(-x-t) d t .
$$

By a theorem of Hardy, (see Zygmund [1935, p. 72]), we have

$$
\int_{0}^{\infty}\left[\frac{1}{x} \int_{0}^{x} f(u) d u\right]^{p} x^{\alpha p} d x \leqq A(\alpha, p) \int_{0}^{\infty} f(x) x^{\alpha p} d x
$$


and

$$
\int_{0}^{\infty}\left[\frac{1}{x} \int_{0}^{x} f(-u) d u\right]^{p} x^{\alpha p} d x \leqq A(\alpha, p) \int_{0}^{\infty} f(-x)^{p} x^{\alpha p} d x
$$

The argument leading up to (10) shows that

$$
\int_{0}^{\infty}\left[\text { l.u.b. } \frac{1}{u} \int_{0}^{u} f(-x-t) d t\right]^{p} x^{\alpha p} d x \leqq A(\alpha, p) \int_{0}^{\infty} f(-x)^{p} x^{\alpha p} d x .
$$

Combining these we obtain

$$
\int_{0}^{\infty} h_{3}(x)^{p} x^{\alpha p} d x \leqq A(\alpha, p) \int_{-\infty}^{\infty} f(x)^{p}|x|^{\alpha p} d x .
$$

From (10), (11), and (12) we see that

$$
\int_{0}^{\infty} h(x)^{p} x^{\alpha p} d x \leqq A(\alpha, p) \int_{-\infty}^{\infty} f(x)^{p}|x|^{\alpha p} d x
$$

Changing $x$ to $-x$ we obtain a similar inequality on $(-\infty, 0)$ and thus $(6)$ is proved.

CoRollaRy 5D. Under the hypotheses of Lemma 5A,

$$
\lim _{y \rightarrow 0} f(x, y)=f(x) \quad \text { a.e. }
$$

and

$$
\underset{y \rightarrow 0}{\lim .{ }^{\alpha, p}} f(x, y)=f(x) .
$$

The first equality is a consequence of the theorem on Cauchy's singular integral, Titchmarsh [1937, p. 30]. The second is immediate using Lemma 5C and Lebesgue's dominated convergence theorem. Similar results for $f^{\sim}(x, y)$ follow from these. We are now ready to prove the main result of this section.

Theorem 5E. Suppose that for $n=1,2, \cdots, N$

(a) $f_{n} \in L^{\alpha, p}(-\infty, \infty), 1<p<\infty,-1 / p<\alpha<1-1 / p$,

(b) that $\alpha_{n}(t)$ is nondecreasing for $t$ in a finite interval $\left[a_{n}, b_{n}\right]$ and that

(c) $L_{n}$ is a subinterval of $\left(0, y_{n}\right]$ of length $L_{n}$.

Then we have

$$
\begin{aligned}
\left\|\left(\sum_{n=1}^{N} \int_{a_{n}}^{b_{n}}\left|S_{0, t}\left[f_{n}\left(x, y_{n}\right)\right]\right|{ }^{2} d \alpha_{n}(t)\right)^{1 / 2}\right\|_{\alpha, p} & \\
& \leqq A(\alpha, p)\left\|\left(\sum_{n=1}^{N} L_{n}^{-1} \int_{L_{n}}\left|f_{n}(x, y)\right|^{2} d y \int_{a_{n}}^{b_{n}} d \alpha_{n}(t)\right)^{1 / 2}\right\|_{\alpha, p} .
\end{aligned}
$$

Proof. We may assume that $f_{n} \in L^{2} \cap L^{\alpha, p}$. Supposing that $t_{n}$ are positive 
reals and that $y_{n}^{\prime} \in\left[0, y_{n}\right]$, we set

$$
\phi_{n}(u)=\left\{\begin{array}{l}
-e^{\left(y_{n^{\prime}}-y_{n}\right) u} \\
0
\end{array}\right.
$$

if $0 \leqq u<t_{n}$, if $u=t_{n}$.

Integrating by parts we obtain

$$
\begin{aligned}
& S_{0, t_{n}}\left[f_{n}\left(x, y_{n}\right)\right]=(2 \pi)^{-1 / 2} \int_{0}^{t_{n}} f_{n}^{\wedge}(u) e^{-i x u-y_{n} u} d u \\
& =(2 \pi)^{-1 / 2} \int_{0}^{t_{n}} e^{\left(y_{n}{ }^{\prime}-y_{n}\right) u} f^{\wedge}(u) e^{-i x u-y_{n}{ }^{\prime} u} d u \\
& =\int_{0}^{t_{n}} e^{\left(y_{n}^{\prime}-y_{n}\right)} d_{u} S_{0, t_{n}}\left[f_{n}\left(x, y_{n}^{\prime}\right)\right] \\
& =e^{\left(y_{n}{ }^{\prime}-y_{n}\right) t_{n} S_{0, t_{n}}}\left[f_{n}\left(x, y_{n}^{\prime}\right)\right]-\int_{0}^{t_{n}} S_{0, u}\left[f_{n}\left(x, y_{n}^{\prime}\right)\right] d e^{\left(y_{n}{ }^{\prime}-y_{n}\right) u} \\
& =\int_{0}^{t_{n}} S_{0, u}\left[f_{n}\left(x, y_{n}^{\prime}\right)\right] d \phi_{n}(u) .
\end{aligned}
$$

Since for every $n$

$$
\int_{0}^{t_{n}}\left|d \phi_{n}(u)\right| \leqq 1
$$

we otbain by Schwarz inequality

$$
\left|S_{0, t_{n}}\left[f_{n}\left(x, y_{n}^{\prime}\right)\right]\right|^{2} \leqq \int_{0}^{t_{n}}\left|S_{0, u}\left[f_{n}\left(x, y_{n}^{\prime}\right)\right]\right|^{2}\left|d \phi_{n}(u)\right|
$$

By Theorem $4 \mathrm{H}$ we conclude that

$$
\begin{aligned}
& \left\|\left(\sum_{n=1}^{N}\left|S_{0, t_{n}}[f(x, y)]\right|^{2}\right)^{1 / 2}\right\| \\
& \quad \leqq\left\|\left(\sum_{n=1}^{N} \int_{0}^{t_{n}}\left|S_{0, u}\left[f_{n}\left(x, y_{n}^{\prime}\right)\right]\right|^{2}\left|d \phi_{n}(u)\right|\right)^{1 / 2}\right\| \\
& \quad \leqq A(\alpha, p)\left\|\left(\sum_{n=1}^{N}\left|f_{n}\left(x, y_{n}^{\prime}\right)\right|^{2}\right)^{1 / 2}\right\| .
\end{aligned}
$$

Let $m$ be a positive integer and let $L_{n}$ be partitioned into $m$ intervals $\left[y_{n, j-1}, y_{n, j}\right], j=1,2, \cdots, m$, of equal length for each $n$. Using (13) we see that

$$
\left\|\left(\sum_{n=1}^{N} \frac{1}{m} \sum_{j=1}^{m}\left|S_{0, t_{n}}\left[f_{n}\left(x, y_{n}\right)\right]\right|^{2}\right)^{1 / 2}\right\| \leqq A\left\|\left(\sum_{n=1}^{N} \frac{1}{m} \sum_{j=1}^{m}\left|f_{n}\left(x, y_{n}^{\prime}\right)\right|^{2}\right)^{1 / 2}\right\| .
$$


On the left we have the norm of the expression

$$
\left(\sum_{n=1}^{N}\left|S_{0, t_{n}}\left[f_{n}\left(x, y_{n}\right)\right]\right|^{2}\right)^{1 / 2}
$$

while on the right we have the norm of an expression which converges to

$$
\left(\sum_{n=1}^{N} L_{n}^{-1} \int_{L_{n}}\left|f_{n}(x, y)\right|^{2} d y\right)^{1 / 2}
$$

as $m \rightarrow \infty$. Since the latter expression is dominated by

$$
\left(\sum_{n=1}^{N} f_{n}^{*}(x)^{2}\right)^{1 / 2}
$$

which is in $L^{\alpha, p}$ by Lemmas $5 \mathrm{C}$ and $4 \mathrm{~F}$, we obtain by Lebesgue's dominated convergence theorem

$$
\left\|\left(\sum_{n=1}^{N}\left|S_{0, t_{n}}\left[f_{n}\left[f_{n}\left(x, y_{n}\right)\right]\right]\right|^{2}\right)^{1 / 2}\right\| \leqq A\left\|\left(\sum_{n=1}^{N} L_{n}^{-1} \int_{L_{n}}\left|f_{n}(x, y)\right|^{2} d y\right)^{1 / 2}\right\| .
$$

The proof may now be completed as in the proof of Theorem $4 \mathrm{H}$.

6 . The $H^{\alpha, p}$ classes. We say that a function $w(z)$, analytic for $I(z)>0$, is in $H^{\alpha, p}$ if

$$
\underset{y>0}{\text { l.u.b. }} \int_{-\infty}^{\infty}|w(x+y i)|^{p}|x+y i|^{a p} d x<\infty .
$$

We now study these classes and their relationship to $L^{\alpha, p}$. We write $H^{p}$ for $H^{0, p}$.

Lemma 6A. If $w(z) \in H^{\alpha, p}, 1<p<\infty,-1 / p<\alpha<1-1 / p$, then there is a function $w(x) \in L^{\alpha, p}(-\infty, \infty)$ such that

$$
\lim _{\nu \rightarrow 0+} w(x+y i)=w(x) \text { a.e. }
$$

and

$$
\underset{y \rightarrow 0+}{\operatorname{li.m.}{ }^{\alpha, p} w(x+y i)}=w(x) .
$$

Proof. In the case $\alpha=0$ this result is well known, Titchmarsh [1937, p. 139]. Since $z^{\alpha} w(z) \in H^{p}$, there is a function $x^{\alpha} w(x) \in L^{p}$ such that

$$
\lim _{y \rightarrow 0+}(x+y i)^{\alpha} w(x+y i)=x^{\alpha} w(x) \text { a.e. }
$$

and

$$
\underset{\nu \rightarrow 0+}{\operatorname{li} . \operatorname{m}}{ }^{p}(x+y i)^{\alpha} w(x+y i)=x^{\alpha} w(x) .
$$


The latter expression may also be written in the form

$$
\lim _{y \rightarrow 0+} \int_{-\infty}^{\infty}\left|(x+y i)^{\alpha} w(x+y i)-x^{\alpha} w(x)\right|^{p} d x=0 .
$$

The relation (2) follows at once from (4). Instead of (5) or $\left(5^{\prime}\right)$ however, we wish to prove (3), that is to show that

$$
\lim _{\nu \rightarrow 0+} \int_{-\infty}^{\infty}\left|x^{\alpha} w(x+y i)-x^{\alpha} w(x)\right|^{p} d x=0 .
$$

We may write

$$
\begin{aligned}
x^{\alpha} w(x+y i)-x^{\alpha} w(x)= & {\left[x^{\alpha} w(x+y i)-(x+y i)^{\alpha} w(x+y i)\right] } \\
& +\left[(x+y i)^{\alpha} w(x+y i)-x^{\alpha} w(x)\right] .
\end{aligned}
$$

Thus to prove (6) it is sufficient in view of $\left(5^{\prime}\right)$ to show that

$$
\lim _{\nu \rightarrow 0+} \int_{-\infty}^{\infty}|w(x+y i)|^{p}\left|x^{\alpha}-(x+y i)^{\alpha}\right| p d x=0 .
$$

In case $\alpha>0$ we write the left side of (7) in the form

$$
\lim _{\nu \rightarrow 0+} \int_{-\infty}^{\infty}\left|(x+y i)^{\alpha} w(x+y i)\right|^{p}\left|x^{\alpha}(x+y i)^{-\alpha}-1\right|^{p} d x
$$

Note that the last term in the integrand is bounded and converges to zero. Setting

$$
w_{\alpha}^{*}(x)=\underset{y>0}{\operatorname{l.u.b}}\left|(x+y i)^{\alpha} w(x+y i)\right|
$$

we see that the first term in the integrand of (8) is dominated by $w_{\alpha}^{*}(x)^{p} \in L^{1}$ by Lemma $5 \mathrm{C}$. Thus (7) holds by the dominated convergence theorem.

The case $\alpha<0$ is proved in almost the same way.

Lemma 6B. If $f(x) \in L^{\alpha, p}(-\infty, \infty), 1<p<\infty,-1 / p<\alpha<1-1 / p$, then $F(z)=f(x, y)+i f^{\sim}(x, y) \in H^{\alpha, p}$ and in fact

$$
\int_{-\infty}^{\infty}|F(x+y i)|^{p}|x+y i|^{\alpha p} d x \leqq A(\alpha, p)\|f(x)\|_{\alpha, p}^{p} .
$$

Proof. In view of Corollary $5 \mathrm{~B}$, it is enough to prove (9). If $\alpha \leqq 0$, then $|x+y i|^{\alpha p} \leqq|x|^{\alpha p}$ and the result is immediate from Minkowski's inequality and Theorem $4 \mathrm{D}$.

If $\alpha>0$, we set 


$$
\begin{aligned}
A(y) & =\int_{-\infty}^{\infty}|F(x+y i)|^{p}|x+y i|^{\alpha p} d x \\
& =\left[\int_{|x|>1}+\int_{-1}^{1}\right]|F(x+y i)|^{p}|x+y i|^{\alpha p} d x=I_{1}+I_{2} .
\end{aligned}
$$

Considering the first integral, we have

$$
\begin{aligned}
I_{1} & \leqq \underset{|x|>1}{\text { l.u.b. }}|(x+y i) / x|^{\alpha p} \int_{-\infty}^{\infty}|F(x+y i)| p|x|^{\alpha p} d x \\
& \leqq A(\alpha, p)\left(1+y^{2}\right)^{\alpha p / 2}\|f(x)\|=O\left(y^{\alpha p}\right) \text { as } y \rightarrow \infty .
\end{aligned}
$$

In considering the second integral we note that by Lemma 5A and Hölder's inequality,

$$
\begin{aligned}
|F(x+y i)| & \leqq\|F(x+u)\|_{\alpha, p}\|P(u, y)\|_{-\alpha, q} \\
& \leqq A(\alpha, p) y^{-1 / p-\alpha}\|f\|_{\alpha, p}
\end{aligned}
$$

and therefore we have

$$
\begin{aligned}
I_{2} & \leqq \underset{|x|<1}{\text {.u.b. }}|x+y i|^{\alpha p} \int_{-1}^{1}|F(x+y i)|^{p} d x \\
& \leqq A(\alpha, p)\left(1+y^{2}\right)^{\alpha p / 2} y^{-1-\alpha p}\|f\|_{\alpha, p} \\
& =O\left(y^{-1}\right)
\end{aligned}
$$$$
\text { as } y \rightarrow \infty \text {. }
$$

It follows that $\log A(y)=o(y)$ as $y \rightarrow \infty$. By the three lines theorem we see that if $0<y_{1}<y_{2}<y_{3}<\infty$, then

$$
\log A\left(y_{2}\right)=\frac{y_{3}-y_{2}}{y_{3}-y_{1}} \log A\left(y_{1}\right)+\frac{y_{2}-y_{1}}{y_{3}-y_{1}} \log A\left(y_{3}\right) .
$$

Letting $y_{3} \rightarrow \infty$, we obtain $\log A\left(y_{2}\right) \leqq \log A\left(y_{1}\right)$; that is

$$
A\left(y_{2}\right) \leqq A\left(y_{1}\right)
$$

and $A(y)$ is a nonincreasing function for $y>0$.

Now let $F_{\alpha}(z)=z^{\alpha} F(z)$. As a function of $x, F_{\alpha}(z) \in L^{p}$ for each $y$ and in fact

$$
F_{\alpha}(x+y i)=\int_{-\infty}^{\infty} F_{\alpha}(u) P(u-x, y) d u .
$$

Therefore by Corollary 5D we have

$$
\underset{y \rightarrow 0+}{\operatorname{l.i.m.}_{y}{ }^{p}} F_{\alpha}(x+y i)=F_{\alpha}(x) .
$$

Since $A(y)=\left\|F_{\alpha}(x+y i)\right\|_{p}^{p}$, we may write the above relation in the form 


$$
\lim _{y \rightarrow 0+} A(y)=A(0) \text {. }
$$

Thus we conclude that for all $y \geqq 0$

$$
A(y) \leqq A(0)=\|F(x)\|_{\alpha, p}^{p} \leqq A(\alpha, p)\|f(x)\|_{\alpha, p}^{p}
$$

We next quote a result due to D. Waterman [1956, p. 173]. The corresponding result for series was first proved by Littlewood and Paley [1937].

Theorem 6C (Waterman). If $w(z) \in H^{p}, 1<p<\infty$, and

$$
g(x \mid w)=\left[\int_{0}^{\infty}\left|w^{\prime}(x+y i)\right|^{2} y d y\right]^{1 / 2},
$$

then

$$
\|g(x \mid w)\|_{p} \leqq A(p)\|w(x)\|_{p}
$$

We now prove a theorem analogous to a theorem of Hirschman [1955a, p. 36] for series. The proof uses methods due to Zygmund [1945, p. 439].

Theorem 6D. If $w(z) \in H^{p}, 1<p<\infty$, and

$$
h(x \mid w)=\left[\int_{0}^{\infty}|w(x+y i) /(x+y i)|^{2} y d y\right]^{1 / 2},
$$

then $\|h(x \mid w)\|_{p} \leqq A(p)\|w(x)\|_{p}$.

Proof. The proof will be given in three parts.

PART 1. The theorem is true for $p=2$. Let $u(x)=\Re[w(x)]$ and

$$
G(t)=\left\{\begin{array}{cc}
0 & \text { if } t \geqq 0, \\
-2 \int_{t}^{0} u^{\wedge}(s) d s & \text { if } t<0 .
\end{array}\right.
$$

We first show that $e^{\nu t} G(t)$ is the Fourier transform of $(i z)^{-1} w(z)$. Writing $w^{\wedge}(t)$ for the Fourier transform of $w(x)$, we see that since $w(x)=u(x)+i u^{\sim}(x)$, Titchmarsh [1937, p. 139],

$$
w^{\wedge}(t)=u^{\wedge}(t)+[i \operatorname{sgn} t] u^{\wedge}(t) i=[1-\operatorname{sgn} t] u^{\wedge}(t) .
$$

Thus $w^{\wedge}(t)$ vanishes for $t>0$ and we may write

$$
w(z)=(2 \pi)^{-1} \int_{-\infty}^{0} 2 u^{\wedge}(t) e^{-i z t} d t .
$$

Moreover, by Schwarz' inequality and Parseval's theorem, we have for $t<0$

$$
|G(t)| \leqq\left[\int_{0}^{-t} d s\right]^{1 / 2}\left[\int_{0}^{-t} 4\left|u^{\wedge}(s)\right|^{2} d s\right]^{1 / 2} \leqq 2|t|^{1 / 2}\|w(x)\|_{2} .
$$


Integrating by parts and using (10) and (11), we obtain for the inverse Fourier transform of $e^{y t} G(t)$

$$
\begin{aligned}
(2 \pi)^{-1 / 2} \int_{-\infty}^{0} e^{y t} G(t) e^{-i x t} d t & \\
& =\left.(2 \pi)^{-1 / 2}(-i z)^{-1} e^{-i z t} G(t)\right|_{t=-\infty} ^{0}+(2 \pi)^{-1 / 2}(i z)^{-1} \int_{-\infty}^{0} 2 u^{\wedge}(t) e^{-i \varepsilon t} d t \\
& =(i z)^{-1} w(z) .
\end{aligned}
$$

Using Parseval's theorem and a theorem of Hardy (see Zygmund [1935, p. 72]), we have

$$
\begin{aligned}
\|h(x \mid w)\|_{2}^{2} & =\int_{0}^{\infty} y d y \int_{-\infty}^{\infty}|w(z) / z|^{2} d x=\int_{0}^{\infty} y d y \int_{-\infty}^{0} e^{2 y t}|G(t)|^{2} d t \\
& =\int_{-\infty}^{0}|G(t) / t|^{2} d t \int_{0}^{\infty} y t^{2} e^{2 y t} d y=\frac{1}{4} \int_{-\infty}^{0}|G(t) / t|^{2} d t \\
& \leqq A \int_{-\infty}^{0}\left|u^{\wedge}(t)\right|^{2} d t=A \|_{w^{\wedge}(t)\left\|_{2}^{2}=A\right\| w(x) \|_{2}^{2} .}
\end{aligned}
$$

PART 2. If the theorem is true for index $p$ and $1<r<p$, then it is true for index $r$. We may suppose that if $w(z) \in H^{r}$ then it has no zeros for $\Im(z)>0$. See Waterman [1956, p. 179]. Setting $v(z)=w(z)^{r^{\prime} p}$, we see that $v(z) \in H^{p}$. We now obtain

$$
h(x \mid w)^{2}=\int_{0}^{\infty}|v(x+y i)|^{2 p / r}|x+y i|^{-2} y d y \leqq v^{*}(x)^{2 p / r-2} h(x \mid v)^{2} .
$$

It follows that

$$
h(x \mid w)^{r} \leqq v^{*}(x)^{p-r} h(x \mid v)^{r} .
$$

Using Hölder's inequality, we conclude that

$$
\begin{gathered}
\|h(x \mid w)\|_{r}^{r} \leqq\left[\int_{-\infty}^{\infty} h(x \mid v)^{p} d x\right]^{r / p}\left[\int_{-\infty}^{\infty} v^{*}(x)^{p} d x\right]^{r / p-1} \\
\leqq A(r)\|v(x)\|_{p}^{p}=A(r)\|w(x)\|_{r}^{r} .
\end{gathered}
$$

In particular we now have the theorem for all $p \leqq 2$.

PART 3. The theorem is true for all $p \geqq 4$ and thus for all $p$. We set $1 / q$ $=1-2 / p$ and observe that

$$
\|h(x \mid w)\|_{p}^{2}=\underset{f}{\text { l.u.b. }} \int_{-\infty}^{\infty} h(x \mid w)^{2} f(x) d x,
$$

where $f$ ranges over all non-negative real valued functions in $L^{q}$ such that $\|f\|_{q} \leqq 1$. 
We set $F(z)=f(x, y)+i f^{\sim}(x, y)$. Since $|w(z) / z|^{2}$ is subharmonic, we have

$$
|w(x+2 y i) /(x+2 y i)| \leqq \int_{-\infty}^{\infty}|w(u+y i) /(u+y i)|{ }^{2} P(u-x, y) d u .
$$

Using Fubini's theorem

$$
\begin{aligned}
\int_{-\infty}^{\infty} h(x \mid w)^{2} f(x) d x & =\int_{0}^{\infty} y d y \int_{-\infty}^{\infty}|w(z) / z|^{2} f(x) d x \\
& =4 \int_{0}^{\infty} y d y \int_{-\infty}^{\infty}|w(x+2 y i) /(x+2 y i)|^{2} f(x) d x .
\end{aligned}
$$

Considering the inner integral we have using (13) and the inequality $f(x, y)$ $\leqq|F(x+y i)|$,

$$
\begin{aligned}
\int_{-\infty}^{\infty} \mid & w(x+2 y i) /(x+2 y i) \mid{ }^{2} f(x) d x \\
& \leqq \int_{-\infty}^{\infty} f(x) d x \int_{-\infty}^{\infty}|w(u+y i) /(u+y i)|^{2} P(u-x, y) d u \\
& \leqq \int_{-\infty}^{\infty}|w(u+y i) /(u+y i)|{ }^{2} f(u, y) d u \\
& \leqq \int_{-\infty}^{\infty}|w(x+y i) /(x+y i)|^{2}|F(x+y i)| d x .
\end{aligned}
$$

Using this together with Schwarz' inequality and Hölder's inequality, we see that

$$
\begin{aligned}
\int_{-\infty}^{\infty} h(x \mid w)^{2} f(x) d x & \leqq 4 \int_{-\infty}^{\infty} w^{*}(x) d x \int_{0}^{\infty}|w(z) / z||F(z) / z| y d y \\
& \leqq 4 \int_{-\infty}^{\infty} w^{*}(x) h(x \mid w) h(x \mid F) d x \\
& \leqq 4\left\|w^{*}(x)\right\|_{p}\|h(x \mid w)\|_{p}\|h(x \mid F)\|_{q} \\
& \leqq A(p)\|w(x)\|_{p}\|h(x \mid w)\|_{p} .
\end{aligned}
$$

We have also used the fact that $q \leqq 2$ and $F(z) \in H^{q}$.

It follows that

$$
\|h(x \mid w)\|_{p}^{2} \leqq A(p)\|h(x \mid w)\|_{p}\|w(x)\|_{p}
$$

If $h(x \mid w) \in L^{p}$, we are done. Otherwise set $w_{\delta}(z)=e^{i \delta z}, \delta>0$. Clearly $w_{\delta}(z)$ $\in H^{p}$ and $\left\|w_{\delta}(x)\right\|_{p} \leqq A(p)\|w(x)\|_{p}$. We show that $h\left(x \mid w_{\delta}\right) \in L^{p}$. Once this is proved the proof may be completed by letting $\delta \rightarrow 0$ and using Fatou's lemma. 
There is no loss of generality in supposing that $w(z-i \epsilon) \in H^{p}$ for $\epsilon>0$. See Waterman [1956, p. 174]. Therefore by Minkowski's inequality for integrals and the Poisson expansion

$$
w(x+y i)=\int_{-\infty}^{\infty} w(x+u-i \epsilon) P(u, y+\epsilon) d u, \quad y \geqq 0,
$$

we obtain

$$
\begin{aligned}
\|w(x+y i)\|_{p} & \leqq \int_{-\infty}^{\infty}\|w(x+u-i \epsilon)\|_{p}|P(u, y+\epsilon)| d u \\
& =\|w(x-i \epsilon)\|_{p}\|P(u, y+\epsilon)\|_{1} \leqq\|w(x-i \epsilon)\|_{p} .
\end{aligned}
$$

If $f(x) \geqq 0$ and $\|f\|_{q} \leqq 1$, we now have

$$
\begin{aligned}
\int_{-\infty}^{\infty} h\left(x \mid w_{\delta}\right)^{2} f(x) d x & =\int_{0}^{\infty} y e^{-2 \delta y} d y \int_{-\infty}^{\infty}|w(z) / z| 2 f(x) d x \\
& \leqq A\|w(x-i \epsilon)\|_{p}^{2} \int_{0}^{\infty} e^{-2 \delta y} d y \int_{-\infty}^{\infty} f(x) P(x, y) d x \\
& \leqq A\|w(x-i \epsilon)\|_{p}^{2} \int_{0}^{\infty} e^{-2 \delta y}\|f(x)\|_{p}\|P(x, y)\|_{p / 2} d y \\
& \leqq A\|w(x-i \epsilon)\|_{p}^{2} \int_{0}^{\infty} e^{-2 \delta y} y^{p / 2-1} d y \\
& \leqq A\|w(x-i \epsilon)\|_{p}^{2}<\infty .
\end{aligned}
$$

It follows that $h\left(x \mid w_{\delta}\right) \in L^{p}$.

Theorem 6E. If $w(z) \in H^{\alpha, p}, 1<p<\infty,-1 / p<\alpha<1-1 / p$, then

$$
\left\|\left(\int_{0}^{\infty}\left|w^{\prime}(x+y i)\right|^{2}|x+y i|^{2 \alpha} y d y\right)^{1 / 2}\right\|_{p} \leqq(\alpha, p)\|w(x)\|_{\alpha, p} .
$$

Proof. We set $v(z)=z^{\alpha} w(z) \in H^{p}$. Since $w^{\prime}(z)=z^{-\alpha} v^{\prime}(z)-\alpha z^{\alpha-1} v(z)$, we have

$$
\left|w^{\prime}(z)\right|^{2} \leqq 2\left|v^{\prime}(z)\right|^{2}|z|^{-2 \alpha}+2|\alpha||v(z)|^{2}|z|^{-2-2 \alpha} .
$$

By Theorems 6C and 6D we conclude that

$$
\begin{aligned}
& \left\|\left(\int_{0}^{\infty}\left|w^{\prime}(x+y i)\right|^{2}|x+y i|^{2 \alpha} y d y\right)^{1 / 2}\right\|_{p} \\
& \quad \leqq A(\alpha)\left[\left\|\left(\int_{0}^{\infty}\left|v^{\prime}(x+y i)\right|^{2} y d y\right)^{1 / 2}\right\|_{p}+\left\|\left(\int_{0}^{\infty}|v(z) / z|^{2} y d y\right)^{1 / 2}\right\|_{p}\right] \\
& \quad \leqq A(\alpha, p) \|_{v(x)\left\|_{p}=A(\alpha, p)\right\| w(x) \|_{\alpha, p} .}
\end{aligned}
$$


7. The decomposition theorem. Let us write $\phi_{n}^{+}(t)$ and $\phi_{n}^{-}(t)$ for the characteristic functions of the intervals $\left[2^{n-1}, 2^{n}\right]$ and $\left[-2^{n},-2^{n-1}\right]$ respectively for each integer $n$. Let $\Delta_{n}^{+}$and $\Delta_{n}^{-}$be the corresponding Fourier multiplier transformations on $L^{\alpha, p}(-\infty, \infty)$. In this section we prove that if $f(x) \in L^{\alpha, p}$, then under the usual restrictions on $\alpha$ and $p$ we have

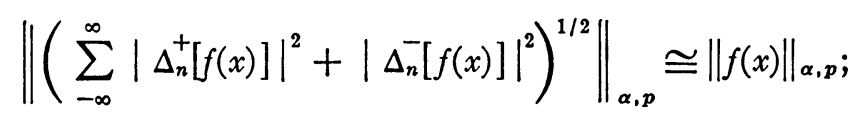

that is, the quotient of these norms is bounded between positive constants depending only on $\alpha$ and $p$. This is called the decomposition theorem. In this connection see Littlewood and Paley [1937] and Hirschman [1955a].

Lemma 7A. If $f \in L^{\alpha, p}, 1<p<\infty,-1 / p<\alpha<1-1 / p$, then

$$
\left\|\left(\sum\left|\Delta_{n}^{+}[f]\right|^{2}+\left|\overline{\Delta_{n}}[f]\right|^{2}\right)^{1 / 2}\right\|_{\alpha, p} \leqq A(\alpha, p)\|f\|_{\alpha, p} .
$$

Proof. There is no loss of generality in supposing that $f \in L^{2} \cap L^{\alpha, p}$ and that $f^{\wedge}(t)=0$ for $t<0$. Then $\Delta_{n}^{-}[f(x)]=0$ for all $x$ and $n$. We write $\Delta_{n}$ for $\Delta_{n}^{+}[f]$.

Differentiating partially with respect to $x$, we obtain

$$
\begin{aligned}
\left(S_{0, t}[f(x, y)]\right)_{x} & =(2 \pi)^{-1 / 2}\left(\int_{0}^{t} f^{\wedge}(u) e^{-y u-i x u} d u\right)_{x} \\
& =(2 \pi)^{-1 / 2} \int_{0}^{t} f^{\wedge}(u)(-i u) e^{-y u-i x u} d u \\
& =S_{0, t}\left[f_{x}(x, y)\right] .
\end{aligned}
$$

Integrating by parts, we see that

$$
\begin{aligned}
\Delta_{n}[f(x)]= & i(2 \pi)^{-1 / 2} \int_{2^{n-1}}^{2^{n}} t^{-1} e^{y t} d S_{0, t}\left[f_{x}(x, y)\right] \\
= & 2^{-n} e^{2^{n}} S_{0,2^{n}}\left[f_{x}(x, y)\right] i-2^{-n+1} e^{2^{n-1}} S_{0,2^{n}}\left[f_{x}(x, y)\right] i \\
& -i \int_{2^{n-1}}^{2^{n}} S_{0, t}\left[f_{x}(x, y)\right] d_{t} t^{-1} e^{y t} .
\end{aligned}
$$

Setting $y=y_{n}=2^{-n}$ for each $n$ and

$$
g_{n}(t)=\left\{\begin{array}{cl}
-t^{-1} e^{y_{n} t} & \text { if } 2^{n-1}<t<2^{n} \\
0 & \text { if } t=2^{n-1} \text { or } 2^{n}
\end{array}\right.
$$

we have

$$
\Delta_{n}[f(x)]=i \int_{2^{n-1}}^{2^{n}} S_{0, t}\left[f_{x}\left(x, y_{n}\right)\right] d g_{n}(t)
$$


Since

$$
\int_{2^{n-1}}^{2^{n}}\left|d g_{n}(t)\right|=2 y_{n} e
$$

it follows from Schwarz' inequality that

$$
\left|\Delta_{n}[f(x)]\right|^{2} \leqq 2 y_{n} e \int_{2^{n-1}}^{2^{n}}\left|S_{0, t}\left[f_{x}\left(x, y_{n}\right)\right]\right|^{2}\left|d g_{n}(t)\right|
$$

We now let $L_{n}$ denote the interval $\left[y_{n+1}, y_{n}\right]$ as well as its length. If we set $F(z)=f(x, y)+i f^{\wedge}(x, y)$, then $\left|f_{x}(x, y)\right| \leqq\left|F^{\prime}(z)\right|$. Since $\alpha \geqq 0,|x|^{\alpha p}$ $\leqq|x+y i|^{\alpha p}$ and therefore using Theorems $5 \mathrm{E}, 6 \mathrm{E}$, and $4 \mathrm{D}$, we conclude that

$$
\begin{aligned}
\left\|\left(\sum_{-\infty}^{\infty}\left|\Delta_{n}[f(x)]\right|^{2}\right)^{1 / 2}\right\|_{\alpha, p} \\
\quad \leqq\left\|\left(\sum_{-\infty}^{\infty} 2 y_{n} e \int_{2^{n-1}}^{2^{n}}\left|S_{0, t}\left[f_{x}\left(x, y_{n}\right)\right]\right|^{2}\left|d g_{n}(t)\right|\right)^{1 / 2}\right\|_{\alpha, p} \\
\quad \leqq A\left\|\left(\sum_{-\infty}^{\infty} y_{n} L_{n}^{-1} \int_{L_{n}}\left|f_{x}(x, y)\right|^{2} d y \int_{2^{n-1}}^{2^{n}}\left|d g_{n}(t)\right|\right)^{1 / 2}\right\|_{\alpha, p} \\
\quad \leqq A\left\|\left(\sum_{-\infty}^{\infty} y_{n+1} \int_{L_{n}}\left|f_{x}(x, y)\right|^{2} d y\right)^{1 / 2}\right\|_{\alpha, p} \\
\quad \leqq A\left\|\left(\sum_{-\infty}^{\infty} \int_{L_{n}}\left|F^{\prime}(x+y i)\right|^{2} y d y\right)^{1 / 2}\right\|_{\alpha, p} \\
\quad \leqq A\left\|\left(\int_{0}^{\infty}\left|F^{\prime}(x+y i)\right|^{2} y d y\right)^{1 / 2}\right\|_{\alpha, p} \\
\quad \leqq A\left\|\left(\int_{0}^{\infty}\left|F^{\prime}(x+y i)\right|^{2}|x+y i|^{2 \alpha} y d y\right)^{1 / 2}\right\|_{p} \\
\quad \leqq A\|F(x)\|_{\alpha, p} \leqq A\|f(x)\|_{\alpha, p} .
\end{aligned}
$$

Lемма 7B. If $f \in L^{\alpha, p}$, where $p$ and $\alpha p$ are non-negative even integers $p \geqq 2, \alpha<1-1 / p$, then

$$
\|f\|_{\alpha, p} \leqq A(\alpha, p)\left\|\left(\sum_{-\infty}^{\infty}\left|\Delta_{n}^{+}[f]\right|^{2}+\left|\widetilde{\Delta_{n}^{-}[f]}\right|^{2}\right)^{1 / 2}\right\|_{\alpha, p} .
$$

Proof. We follow the methods of Littlewood and Paley [1937, p. 84] and Hirschman [1955a, p. 46] and consider the typical case $p=6, \alpha p=4$. There is no loss of generality in supposing that $f \in L^{2} \cap L^{\alpha, p}$ and that $f^{\wedge}(t)=0$ for $t<0$. We write $\Delta_{n}$ for $\Delta_{n}^{+}[f]$.

We set 


$$
F_{N}(x)=\sum_{-N}^{N} \Delta_{n}(x) \quad \text { and } \quad G_{N}(x)^{2}=\sum_{-N}^{N}\left|\Delta_{n}(x)\right|^{2}
$$

for $N=1,2, \cdots$. We split each of these sums into $h$ parts as follows: for $i=0,1, \cdots, h-1$ set

$$
F_{N, i}(x)=\sum_{n} \Delta_{n}(x) \text { and } G_{N, i}(x)^{2}=\sum_{n}\left|\Delta_{n}(x)\right|^{2}
$$

where $n$ ranges over all integers congruent to $i$ modulo $h$ between $-N$ and $N$. Then there are constants $A_{1}, A_{2}, A_{3}$, and $A_{4}$ such that

$$
\begin{aligned}
\left|F_{N, i}\right|= & A_{1} \sum\left|\Delta_{k}\right|^{2} \Delta_{m} \bar{\Delta}_{m^{\prime}} \Delta_{n} \bar{\Delta}_{n^{\prime}}+A_{2} \sum\left|\Delta_{k}\right|^{2}\left|\Delta_{m}\right|^{2} \Delta_{n} \bar{\Delta}_{n^{\prime}} \\
& +A_{3} \sum\left|\Delta_{k}\right|^{4} \Delta_{n} \Delta_{n^{\prime}}+A_{4} \sum\left|\Delta_{k}\right|^{2}\left|\Delta_{m}\right|^{2}\left|\Delta_{n}\right|^{2} \\
& +\sum^{*} \Delta_{k} \bar{\Delta}_{k^{\prime}} \Delta_{m} \bar{\Delta}_{m^{\prime}} \Delta_{n} \bar{\Delta}_{n^{\prime}},
\end{aligned}
$$

where in each summation all subscripts range through values congruent to $i \bmod h$ and where ${ }^{*}$ denotes a summation in which no one of $k, m, n$ equal any of $k^{\prime}, m^{\prime}, n^{\prime}$.

For $j=1,2, \cdots$, we set $E_{j}=\left\{v: j^{-2} \leqq|v| \leqq j^{-1}\right\}$ and

$$
w_{j}(x)=\frac{1}{2} j \int_{E_{j}}\left|1-e^{i x v}\right|{ }^{4}|v|^{-4} d v .
$$

It is easy to see that $w_{j}(x)$ is less than $|x|^{4}$ and approaches it as $j \rightarrow \infty$. Using the inequality

$$
\sum\left|\Delta_{k}\right|^{4} \leqq\left(\left|\sum \Delta_{k}\right|^{2}\right)^{2}
$$

we have

$$
\begin{aligned}
\int_{-\infty}^{\infty} \mid & F_{N, j}(x) \mid{ }^{6} w_{j}(x) d x \\
\leq & \left.\int_{-\infty}^{\infty}\left|\sum\right| \Delta_{k}(x)\right|^{2} \Delta_{m}(x) \bar{\Delta}_{m^{\prime}}(x) \Delta_{n}(x) \bar{\Delta}_{n^{\prime}}(x) \mid w_{j}(x) d x \\
& +\left.\int_{-\infty}^{\infty}\left|\sum\right| \Delta_{k}(x)\right|^{2}\left|\Delta_{m}(x)\right|^{2} \Delta_{n}(x) \bar{\Delta}_{n^{\prime}}(x) \mid w_{j}(x) d x \\
& +\int_{-\infty}^{\infty} \sum\left|\Delta_{k}(x)\right|^{2}\left|\Delta_{m}(x)\right|^{2}\left|\Delta_{n}(x)\right|{ }^{2} w_{j}(x) d x \\
& +\left|\int_{-\infty}^{\infty} \sum^{*} \Delta_{k}(x) \bar{\Delta}_{k^{\prime}}(x) \Delta_{m}(x) \bar{\Delta}_{m^{\prime}}(x) \Delta_{n}(x) \bar{\Delta}_{n^{\prime}}(x) w_{j}(x) d x\right| .
\end{aligned}
$$

Let $J^{*}$ denote the last integral and consider a single term of the sum. By Fubini's theorem we have 


$$
\begin{aligned}
\frac{j}{2} \int_{-\infty}^{\infty} \Delta_{k} \bar{\Delta}_{k^{\prime}} \Delta_{m} \bar{\Delta}_{m^{\prime}} \Delta_{n} \bar{\Delta}_{m^{\prime}} w_{j} d x & \\
& =\frac{j}{2} \int_{E_{j}}|v|^{-4} d v \int_{-\infty}^{\infty} \Delta_{k} \bar{\Delta}_{k^{\prime}} \Delta_{m} \bar{\Delta}_{m^{\prime}} \Delta_{n} \bar{\Delta}_{n^{\prime}}\left|1-e^{i x v}\right|^{4} d x .
\end{aligned}
$$

The inner integral on the right is a combination of terms of the form

$$
K_{r}(v)=\int_{-\infty}^{\infty} \bar{\Delta}_{k} \Delta_{k^{\prime}} \bar{\Delta}_{m} \Delta_{m} \Delta_{n} \bar{\Delta}_{n^{\prime}} e^{i x v r} d x
$$

where $r=-2,-1,0,1,2$. Writing $\Delta^{\wedge}$ for the Fourier transform of $\Delta$, we obtain the convolution

$$
K_{r}(v)=\left[\Delta_{k} * \bar{\Delta}_{k^{\prime}} * \Delta_{m} * \bar{\Delta}_{\hat{m}^{\prime}} * \Delta_{n}^{\hat{n}} * \bar{\Delta}_{\hat{n}^{\prime}}\right](r v) .
$$

Since $\hat{\Delta_{n}}(t)=0$ for all $t$ outside $\left[2^{n-1}, 2^{n}\right]$, we have for large enough $h$ and small enough $v$ (that is for large enough $j$ ) $K_{r}(v)=0$ for all $v \in E_{j}$. Thus $J^{*}$ vanishes for large enough $h$ and $j$.

Under these conditions we obtain, using Schwarz' inequality,

$$
\begin{aligned}
\int_{-\infty}^{\infty}\left|F_{N, i}\right|{ }^{6} w_{j} d x \leqq & A\left[\int_{-\infty}^{\infty} G_{N, i}^{2}\left|F_{N, i}\right|^{4}|x|^{4} d x\right. \\
& \left.+\int_{-\infty}^{\infty} G_{N, i}^{4}\left|F_{N, i}\right|^{2}|x|^{4} d x+\int_{-\infty}^{\infty} G_{N, i}^{6}|x|^{4} d x\right] \\
\leqq & A\left[\left\|F_{N, i}\right\| 4\left\|G_{N, i}\right\|^{2}+\left\|F_{N, i}\right\|^{2}\left\|G_{N, i}\right\|^{4}+\left\|G_{N, i}\right\|^{6}\right] .
\end{aligned}
$$

Letting $j \rightarrow \infty$ and using Fatou's lemma, we have the same inequality as just above with $\left\|F_{N, i}\right\|^{6}$ on the right. Since $F_{N, i}(x)$ is a multiplier transform to which Theorem $4 \mathrm{D}$ applies, it follows that $\left\|F_{N, i}\right\|$ is finite. Therefore we have $\left\|F_{N, i}\right\| \leqq\left\|G_{N, i}\right\|$. Combining $h$ such inequalities, we see that

$$
\left\|F_{N}\right\| \leqq A(p)\left\|G_{N}\right\| \leqq A(p)\left\|\left(\sum\left|\Delta_{n}\right|^{2}\right)^{1 / 2}\right\| .
$$

Letting $N \rightarrow \infty$, the proof is completed.

Suppose that $\epsilon_{n}^{+}$and $\epsilon_{n}^{-}$are sequences each of whose terms is 1 or -1 . Set $\epsilon=\left(\epsilon_{n}^{+}, \epsilon_{n}^{-}\right)$and

$$
\phi_{\epsilon}(t)= \begin{cases}\epsilon_{n}^{+} & \text {if } t \in\left[2^{n-1}, 2^{n}\right], \\ \epsilon_{n} & \text { if } t \in\left[-2^{n},-2^{n-1}\right] .\end{cases}
$$

We write $T_{\epsilon}$ for the corresponding multiplier transformation and note that for every $f \in L^{\alpha, p}$ we have

$$
T_{\epsilon}[f(x)]=\sum_{-\infty}^{\infty}+\epsilon_{n}^{+} \Delta_{n}^{+}[f(x)]+\overline{\epsilon_{n} \Delta_{n}^{-}}[f(x)]
$$


We also write $\|T\|_{\alpha, p}$ for the norm of a bounded linear transformation $T$ of $L^{\alpha, p}$ into itself.

Lemma 7C. Suppose that $1<p<\infty$, and $-1 / p<\alpha<1-1 / p$. Then the following are equivalent:

(i) there is a constant $A$ such that $\left\|T_{\epsilon}\right\|_{\alpha, p} \leqq A$ for all $\epsilon$;

(ii) $\|f\|_{\alpha, p} \cong\left\|\left(\sum_{-\infty}^{\infty}\left|\Delta_{n}^{+}[f]\right|^{2}+\left|\Delta_{n}^{-}[f]\right|^{2}\right)^{1 / 2}\right\|_{\alpha, p}$ for all $f \in L^{\alpha, p}$.

The proof for Fourier series, Littlewood and Paley [1937, p. 86], needs only trivial changes to be applied here.

Lemma 7D. If $1<p<\infty,-1 / p<\alpha_{1}, \alpha_{2}<1-1 / p,\|T\|_{\alpha_{j}, p}<\infty, j=1,2$, and $\gamma=(1-\theta) \alpha_{1}+\theta \alpha_{2}$ for $0 \leqq \theta \leqq 1$, then

$$
\|T\|_{\gamma, p} \leqq\|T\|_{\alpha_{1}, p}^{1-\theta}\|T\|_{\alpha_{2}, p}^{\theta}
$$

This result, a demonstration of which is given by Hirschman [1956], is essentially contained in the Riesz-Thorin convexity theorem.

Lemma 7E. If $1<p<\infty,-1 / p<\alpha<1-1 / p, 1 / q=1-1 / p$, and $T$ is a multiplier transformation on $L^{\alpha, p}$ such that $\|T\|_{\alpha, p}<\infty$, then $\|T\|_{-\alpha, q}<\infty$.

Proof. It is sufficient to prove this on $L^{2} \cap L^{\alpha, p}$. We may also suppose that $f$ and $\phi$ are real. Suppose $g \in L^{2} \cap L^{\alpha, p}$ and $g$ is real valued. Using Plancherel's theorem, we obtain

$$
\int_{-\infty}^{\infty} T[f(x)] g(x) d x=\int_{-\infty}^{\infty} \phi(t) f^{\wedge}(t) g^{\wedge}(t) d t=\int_{-\infty}^{\infty} f(x) T[g(x)] d x .
$$

If, in addition, $\|g\|_{\alpha, p} \leqq 1$, we have by Hölder's inequality

$$
\|T[f]\|_{-\alpha, q}=1 . \text { u.b. } \int_{-\infty}^{\infty} f(x) T[g(x)] d x \leqq\|f\|_{-\alpha, q}\|T g\|_{\alpha, p} \leqq\|T\|_{\alpha, p}\|f\|_{-\alpha, q} .
$$

Therefore $\|T\|_{-\alpha, q} \leqq\|T\|_{\alpha, p}<\infty$. By symmetry we even have equality.

Theorem 7F. (Decomposition theorem). If $f \in L^{\alpha, p}(-\infty, \infty)$, for $1<p<\infty,-1 / p<\alpha<1-1 / p$, then

$$
\|f\|_{\alpha, p} \cong\left\|\left(\sum_{-\infty}^{\infty}\left|\Delta_{n}^{+}[f]\right|^{2}+\left|\overline{\Delta_{n}}[f]\right|^{2}\right)^{1 / 2}\right\|_{\alpha, p} .
$$

Proof. Let $p^{\prime}=2 m$ be an even integer greater than $p$. Let $\alpha_{1}=0$ and $\alpha_{2}=1-1 / m$. By Lemmas 7A and 7B we obtain for $j=1,2$,

$$
\|f\|_{\alpha_{j}, p^{\prime}} \cong\left\|\left(\sum\left|\Delta_{n}^{+}[f]\right|^{2}+\left|\overline{\Delta_{n}}[f]\right|^{2}\right)^{1 / 2}\right\|_{\alpha_{j}, p^{\prime}} .
$$

By Lemma $7 \mathrm{C}$ there is a constant $A$ such that for all $\epsilon,\left\|T_{\epsilon}\right\|_{\alpha_{j}, p^{\prime}} \leqq A$. Thus by Lemma $7 \mathrm{D}$ we have for all $\alpha^{\prime}$ such that $0 \leqq \alpha^{\prime} \leqq 1-1 / m$ 


$$
\left\|T_{\epsilon}\right\|_{\alpha^{\prime}, p^{\prime}} \leqq A .
$$

By Lemma $7 \mathrm{E}$ we obtain for $1 / q^{\prime}=1-1 / p^{\prime}$

$$
\left\|T_{\epsilon}\right\|_{-\alpha^{\prime}, q^{\prime}} \leqq A .
$$

There is a number $\theta^{\prime}, 0 \leqq \theta^{\prime} \leqq 1$, such that

$$
1 / p=\theta^{\prime} / p+\left(1-\theta^{\prime}\right) / q^{\prime} .
$$

Numbers $\alpha^{\prime}$ and $\beta^{\prime}$ can now be found so that

$$
-1+1 / m \leqq \beta^{\prime} \leqq 0 \leqq \alpha^{\prime} \leqq 1-1 / m
$$

and for the given $\alpha$

$$
\alpha=\theta^{\prime} \alpha^{\prime}+\left(1-\theta^{\prime}\right) \beta^{\prime}
$$

when $m$ is sufficiently large. Since for every sequence $\epsilon$

$$
\left\|T_{\epsilon}\right\|_{\boldsymbol{\alpha}^{\prime}, p^{\prime}} \leqq A \text { and }\left\|T_{\epsilon}\right\|_{\beta^{\prime}, p^{\prime}} \leqq A,
$$

we obtain by the Riesz interpolation theorem

$$
\left\|T_{\epsilon}\right\|_{\alpha, p} \leqq A
$$

for every $\epsilon$. Another application of $9 \mathrm{C}$ completes the proof.

8. The main theorem. We conclude by proving the theorem on the boundedness of the Fourier multiplier transformation and by extending this result to Hankel multiplier transformations. We say that $\phi(t),-\infty<t<\infty$, is in $M(C)$ if

$$
\begin{array}{cr}
|\phi(t)| \leqq C, & -\infty<t<\infty, \\
\int_{2^{n-1}}^{2^{n}}|d \phi(t)| \leqq C, & n=0, \pm 1, \pm 2, \cdots,
\end{array}
$$

and

$$
\int_{-2^{n}}^{-2^{n-1}}|d \phi(t)| \leqq C, \quad n=0, \pm 1, \pm 2, \cdots
$$

If $\phi(t)$ is defined for $0 \leqq t<\infty$, we require only (2) and (1) on this range.

Theorem 8A. If $f \in L^{\alpha, p}(-\infty, \infty), 1<p<\infty,-1 / p<\alpha<1-1 / p$, and $\phi \in M(C)$, then the associated Fourier multiplier transformation $T$ is defined on $L^{\alpha, p}$ and

$$
\|T[f]\|_{\alpha, p} \leqq A(\alpha, p) C\|f\|_{\alpha, p} .
$$

Proof. We may suppose that $f \in L^{2} \cap L^{\alpha, p}$ and that $f^{\wedge}(t)=0$ for $t<0$. We write $\Delta_{n}(x)$ for $\Delta_{n}^{+}[f(x)]$. Let 


$$
D_{n}(x)=(2 \pi)^{-1 / 2} \int_{2^{n-1}}^{2^{n}} \phi(t) f^{\wedge}(t) e^{-i x t} d t
$$

and

$$
F_{N}(x)=(2 \pi)^{-1 / 2} \int_{2^{-N+1}}^{2 N} \phi(t) f^{\wedge}(t) e^{-i x t} d t=\sum_{-N}^{N} D_{n}(x) .
$$

It is enough to prove that

$$
\left\|F_{N}\right\|_{\alpha, p} \leqq A C\|f\|_{\alpha, p}
$$

By the decomposition theorem

$$
\left\|F_{N}\right\|_{\alpha, p} \leqq A\left\|\left(\sum_{-N}^{N}\left|D_{n}\right|^{2}\right)^{1 / 2}\right\|_{\alpha, p} .
$$

For $t \in\left[2^{n-1}, 2^{n}\right]$ we consider

$$
S_{0, t}\left[\Delta_{n}(x)\right]=(2 \pi)^{-1 / 2} \int_{0}^{t} \hat{\Delta_{n}}(u) e^{-i x u} d u=(2 \pi)^{-1 / 2} \int_{2^{n-1}}^{t} f^{\wedge}(t) e^{-i x u} d u .
$$

Integrating by parts, we obtain

$$
\begin{aligned}
D_{n}(x) & =\int_{2^{n-1}}^{2^{n}} \phi(t) d_{t} S_{0, t}\left[\Delta_{n}(x)\right]=\phi\left(2^{n}\right) S_{0,2} n\left[\Delta_{n}(x)\right]-\int_{2^{n-1}}^{2^{n}} S_{0, t}\left[\Delta_{n}(x)\right] d \phi(t) \\
& =\int_{2^{n-1}}^{2^{n}} S_{0, t}\left[\Delta_{n}(x)\right] d \phi_{n}(t)
\end{aligned}
$$

where $\phi_{n}(t)=-\phi(t)$ when $2^{n-1} \leqq t<2^{n}$ and $\phi_{n}\left(2^{n}\right)=0$.

By Schwarz' inequality, we see that

$$
\left|D_{n}(x)\right|^{2} \leqq 2 C \int_{2^{n-1}}^{2^{n}}\left|S_{0, t}\left[\Delta_{n}(x)\right]\right|^{2}\left|d \phi_{n}(t)\right|
$$

since by (2) we have

$$
\int_{2^{n-1}}^{2^{n}}\left|d \phi_{n}(t)\right| \leqq 2 C
$$

for all $n$. By (6), (7), Theorem $4 \mathrm{H},(8)$ and the decomposition theorem we obtain

$$
\begin{aligned}
\left\|F_{N}\right\|_{\alpha, p} & \leqq A C^{1 / 2}\left\|\left(\sum_{-N}^{N} \int_{2^{n-1}}^{2^{n}}\left|S_{0, t}\left[\Delta_{n}[f]\right]\right|^{2}\left|d \phi_{n}(t)\right|\right)^{1 / 2}\right\|_{\alpha, p} \\
& \leqq A C\left\|\left(\sum_{-\infty}^{\infty}\left|\Delta_{n}[f]\right|^{2}\right)^{1 / 2}\right\|_{\alpha, p} \leqq A C\|f\|_{\alpha, p},
\end{aligned}
$$

completing the proof. 
We now extend this result to Hankel multiplier transformations. We find it more natural here to use the usual definition for the Hankel transform rather than that used in the first three sections. As before it is sufficient to define the transformation on a dense subspace. In particular if $f \in L^{\alpha, p}(0, \infty)$ $\cap L^{2}(0, \infty)$ and $\nu \geqq-1 / 2$, we set

$$
F_{\nu}(t)=\int_{0}^{\infty} f(x) J_{\nu}(x t)(x t)^{1 / 2} d x
$$

the integral being a limit in the mean of order 2 of partial integrals. Note that $F_{-1 / 2}$ and $F_{1 / 2}$ are simply the cosine and sine transforms of $f$. Therefore if we extend $f$ to be an even or an odd function on $(-\infty, \infty)$ and apply Theorem $8 \mathrm{~A}$, we obtain the following:

Corollary 8B. If $f \in L^{\alpha, p}(0, \infty), 1<p<\infty,-1 / p<\alpha<1-1 / p$, and $\phi \in M(C)$, then the associated Hankel multiplier transformations $T_{\nu}$ of orders $\nu=-1 / 2$ and $+1 / 2$ exist and

$$
\left\|T_{\nu}[f]\right\|_{\alpha, p} \leqq A(\alpha, p, \nu) C\|f\|_{\alpha, p} .
$$

Lemma 8C. If $\nu, \mu>-1 / 2$, and $g(s)$ is a function on $(0, \infty)$ with Hankel transforms $G_{\nu}(x)$ and $G_{\mu}(x)$ lying in $L^{\alpha, p}(0, \infty)$ and such that

$$
\int_{0}^{\infty} s^{-1 / p-\alpha}|g(s)| d s<\infty,
$$

then

$$
\left\|G_{\nu}(x)\right\|_{\alpha, p} \cong\left\|G_{\mu}(x)\right\|_{\alpha, p}
$$

Proof. We are given

$$
G_{\nu}(x)=\int_{0}^{\infty} g(s) J_{\nu}(x s)(x s)^{1 / 2} d s
$$

and a similar expression for $G_{\mu}(x)$. Setting $x=e^{y}$ and $s=e^{-u}$, we obtain

$$
G_{\nu}\left(e^{y}\right)=\int_{-\infty}^{\infty} g\left(e^{-u}\right) e^{-u} J_{\nu}\left(e^{y-u}\right) e^{(y-u) / 2} d u .
$$

Setting $r=\alpha+1 / p$ and multiplying both sides by $e^{r y}$, we obtain

$$
e^{r \nu} G_{\nu}\left(e^{y}\right)=\int_{-\infty}^{\infty} g\left(e^{-u}\right) e^{-u(1-r)} J_{\nu}\left(e^{y-u)} e^{(r+1 / 2)(y-u)} d u .\right.
$$

On the right we have the convolution of two functions $h(y)=g\left(e^{y}\right) e^{-(1-r) v}$ and $j_{\nu}(y)=J_{\nu}\left(e^{y}\right) e^{(r+1 / 2) y}$. We set $E_{\nu}(y)=e^{r y} G_{\nu}\left(e^{y}\right)$ and let the caret denote the Fourier transform. We show that 


$$
E_{\hat{v}}(t)=h^{\wedge}(t) j \hat{v}(t) .
$$

Note that although $h \in L^{1}(-\infty, \infty)$ by (12), $j_{v}$ need not and thus (15) is not immediate. Considering the partial integral of $E_{\nu}^{\hat{\nu}}$ we obtain

$$
\begin{aligned}
(2 \pi)^{-1 / 2} \int_{-a}^{a} E_{\nu}(y) e^{i t y} d y & =(2 \pi)^{-1} \int_{-a}^{a} e^{i t y} d y \int_{-\infty}^{\infty} h(u) j_{\nu}(y-u) d u \\
& =(2 \pi)^{-1} \int_{-\infty}^{\infty} h(u) d u \int_{-a}^{a} j_{\nu}(y-u) e^{i t y} d y \\
& =(2 \pi)^{-1} \int_{-\infty}^{\infty} h(u) d u \int_{-a-u}^{a-u} j_{\nu}(y) e^{i t(u+y)} d u \\
& =(2 \pi)^{-1 / 2} \int_{-\infty}^{\infty} h(u) e^{i t u} I_{a}(u, t) d u
\end{aligned}
$$

where

$$
I_{a}(u, t)=(2 \pi)^{-1 / 2} \int_{-a-u}^{a-u} j_{\nu}(y) e^{i t y} d y .
$$

By the Weber-Schafheitlin formula, Watson [1944, pp. 391-392] $j_{\nu}^{\wedge}(t)$ $=\lim _{a \rightarrow \infty} I_{a}(u, t)$ exists and has the value

$$
\frac{2^{r-1 / 2+i t} \Gamma(\nu / 2+r / 2+1 / 4+i t / 2)}{(2 \pi)^{1 / 2} \Gamma(\nu / 2-r / 2+3 / 4-i t / 2)} .
$$

Since $0<r<1$, the real part in the arguments of each of the gamma functions is positive and bounded away from zero. Thus $\hat{j_{\nu}}(t)$ is bounded. Moreover $I_{a}(u, t)$ converges boundedly to $\hat{j_{v}}(t)$. Therefore we have (15) by dominated convergence.

A similar expression for $\mu$ holds. Upon dividing the latter into the former, we have

$$
E_{\nu}(t) / E_{\mu}(t)=j \hat{\nu}(t) / j \hat{\mu}(t)=\phi_{\nu, \mu}(t)=\phi(t)
$$

where by (17)

$$
\phi(t)=\frac{\Gamma(\nu / 2+r / 2+1 / 4+i t / 2) \Gamma(\mu / 2-r / 2+3 / 4-i t / 2)}{\Gamma(\mu / 2+r / 2+1 / 4+i t / 2) \Gamma(\nu / 2-r / 2+3 / 4-i t / 2)} .
$$

It follows that $\hat{E_{\nu}}(t)=\phi(t) \hat{E_{\mu}}(t)$. We wish to apply Theorem $4 \mathrm{D}$ and begin by observing that $\phi$ is bounded near zero. Using (18) and a formula from Erdélyi [1953, vol. 1, p. 47] we obtain

$$
\begin{aligned}
& \phi(t)=(i t / 2)^{(\nu-\mu) / 2}\left[1+O\left(t^{-1}\right)\right](-i t / 2)^{(\mu-\nu) / 2}\left[1+O\left(t^{-1}\right)\right] \\
& =e^{i \pi(\nu-\mu) \operatorname{sgn} t / 2}\left[1+O\left(t^{-1}\right)\right] \\
& \text { as }|t| \rightarrow \infty \text {. }
\end{aligned}
$$

It follows that $\phi$ is bounded everywhere. 
We next show that

$$
\int_{-\infty}^{\infty}|d \phi(t)|<\infty
$$

It is easily seen that $\phi$ has finite variation on any finite interval. It is not hard to justify term by term differentiation in (19) and thus obtain $\phi^{\prime}(t)$ $=O\left(t^{-2}\right)$ as $|t| \rightarrow \infty$. It follows that

$$
\int_{|t|>a}|d \phi(t)| \leqq \int_{|t|>a} t^{-2} d t<\infty .
$$

We now have (20) and thus the postulates of Theorem 4D.

We now conclude that

$$
\left\|E_{p}(y)\right\|_{p} \leqq A\left\|E_{\mu}(y)\right\|_{p} .
$$

Making the change in variable $e^{y}=x$, we have

$$
\left\|E_{\nu}(y)\right\|_{p}=\left\|e^{r \nu} G_{\nu}\left(e^{y}\right)\right\|_{p}=\left\|x^{\alpha} G_{\nu}(x)\right\|=\left\|G_{\nu}(x)\right\|_{\alpha, p} .
$$

The same holds with $\mu$ instead of $\nu$. We conclude from (22) that

$$
\left\|G_{\nu}(x)\right\|_{\alpha, p} \leqq A\left\|G_{\mu}(x)\right\|_{\alpha, p} .
$$

Since this holds also with $\nu$ and $\mu$ interchanged, we are done.

Theorem 8D. (The main theorem). If $f \in L^{\alpha, p}(0, \infty)$ for $1<p<\infty$ and $-1 / p<\alpha<1-1 / p$ and if $\phi \in M(C)$ and if $\nu \geqq-1 / 2$, then the Hankel multiplier transformation $T_{\nu}$ associated with $\phi$ exists and

$$
\left\|T_{\nu}[f]\right\|_{\alpha, p} \leqq A(\alpha, \nu, p) C\|\|_{\alpha, p} .
$$

Proof. Suppose that $f$ is a step function which vanishes outside a compact subinterval of $(0, \infty)$. Since the set of all such functions is dense in $L^{\alpha, p}$, this involves no loss of generality. Let $g(s)$ be the Hankel transform of $f(x)$ of order $\nu$ and $G_{1 / 2}(x)$ be the Hankel transform of $g(s)$ of order $1 / 2$. Since $f \in L^{1}$ and is of bounded variation, the Hankel transform of order $\nu$ of $g(s)$ is equal to $f(x)$ almost everywhere.

Now $f(x)$ is a linear combination of characteristic functions of finite intervals. Let $f_{*}(x)$ be such a function for $[a, b]$. Then its Hankel transform is

$$
g_{*}(s)=\int_{a}^{b} J_{\nu}(x s)(x s)^{1 / 2} d x .
$$

Since $J_{\nu}(z) z^{1 / 2}=A_{1} \sin z+A_{2} \cos z+O\left(z^{-1}\right)$ as $z \rightarrow \infty$, it follows that $g_{*}(s)$ $=O\left(s^{-1}\right)$ as $s \rightarrow \infty$. Clearly $g_{*}(s)=O(1)$ as $s \rightarrow 0$. It follows that

$$
\int_{0}^{\infty}\left|g_{*}(s)\right| s^{-1 / p-\alpha} d s \leqq A\left[\int_{0}^{1} s^{-1 / p-\alpha} d s+\int_{1}^{\infty} s^{-1 / p-\alpha-1} d s\right]<\infty,
$$


since $0<1 / p+\alpha<1$. Therefore we have

$$
\int_{0}^{\infty}|g(s)| s^{-1 / p-\alpha} d s<\infty .
$$

We now apply Lemma $8 \mathrm{C}$ to obtain

$$
\|f(x)\|_{\alpha, p} \cong\left\|G_{1 / 2}(x)\right\|_{\alpha, p} .
$$

Next let $T_{1 / 2}\left[G_{1 / 2}(x)\right]$ and $T_{\nu}[f(x)]$ denote the Hankel transforms of orders $1 / 2$ and $\nu$ respectively of $\phi(s) g(s)$. Since $\phi$ is bounded, $\phi g$ satisfies the hypotheses of Lemma $8 \mathrm{C}$ and we have

$$
\left\|T_{1 / 2}\left[G_{1 / 2}\right]\right\|_{\alpha, p} \cong\left\|T_{\nu}[f]\right\|_{\alpha, p} .
$$

By Corollary $8 \mathrm{~B}$ we also have

$$
\left\|T_{1 / 2}\left[G_{1 / 2}\right]\right\|_{\alpha, p} \leqq A(\alpha, p) C\left\|G_{1 / 2}\right\|_{\alpha, p} .
$$

Combining the last three, we obtain (25).

\section{REFERENCES}

S. BOCHNER

1954. Positive zonal function on spheres, Proc. Nat. Acad. Sci. U.S.A. vol. 40, pp. 11411147.

S. Bochner and K. Chandrasekharan

1949. Fourier transforms, Princeton.

J. Delsarte

1938. Sur une extension de la formula de Taylor, J. Math. Pures Appl. vol. 171, pp. 213231.

A. Erdélyi, W. Magnus, F. Oberhettinger and F. Tricomi

1953. Higher transcendental functions, vol. 1, New York.

G. HARDY and J. LiTTLEWOOD

1936. Some more theorems concerning Fourier series, Duke Math. J. vol. 2, pp. 354-382.

G. Hardy, J. Littlewood and G. Polya

1952. Inequalities, Cambridge.

I. I. Hirschman, JR.

1955a. The Decomposition of Walsh and Fourier series, Memoirs Amer. Math. Soc., no. 15, $65 \mathrm{pp}$.

1955b. Weighted quadratic norms and Legendre polynomials, Canad. J. Math. vol. 7, pp. $462-482$.

1956a. A note on orthogonal systems, Pacific J. Math. vol. 6, pp. 47-56.

1956b. Harmonic analysis and ultraspherical polynomials, Symposium on Harmonic Analysis and Related Integral Transforms held at Cornell University, vol. 1.

1957. Projections associated with Jacobi polynomials, Proc. Amer. Math. Soc. vol. 8, pp. 286-290.

I. I. Hirschman JR. And R. Askey

1959. Weighted quadratic norms and ultraspherical polynomials, Trans. Amer. Math. Soc. vol. 91, pp. 294-313.

B. M. LEvitan

1949. The application of generalized displacement operators to linear differential equations of second order, Amer. Math. Soc. Translations, no. 59. 
J. Littlewood and R. E. A. C. Paley

1937. Theorems on Fourier series and power series II, Proc. London Math. Soc. vol. 42, pp. 52-89.

L. H. Loomis

1953. Abstract harmonic analysis, New York.

J. MARCINKIEWICZ

1939. Sur les multiplicateurs des séries de Fourier, Studia Math. vol. 8, pp. 78-91.

R. E. A. C. Paley

1931. Some theorems on orthogonal functions, Studia Math. vol. 3, pp. 226-239.

H. Pollard

1947. The mean convergence of orthogonal series, Trans. Amer. Math. Soc. vol. 62, pp. 387-403.

1948. The mean convergence of orthogonal series. II, Trans. Amer. Math. Soc. vol. 63, pp. 355-367.

1949. The mean convergence of orthogonal series. III, Duke Math. J. vol. 16, pp. 189-191.

M. RIESZ

1923. Über eine Verallgemeinerung der Parsevalschen Formel, Math. Z. vol. 18, pp. 117-124.

1927. Sur la formule d'inversion de Fourier, Acta Sci. Math. Szeged vol. 3, pp. 235-241.

I. N. SNEDDON

1951. Fourier transforms, New York.

S. B. STEČKIN

1950. On bilinear forms, Dokl. Akad. Nauk SSSR, N.S. vol. 71, pp. 237-240.

E. C. Titchmarsh

1922. Hankel transforms, Proc. London Math. Soc. vol. 45, pp. 458-474.

1937. The theory of Fourier integrals, Oxford.

D. WATERMAN

1956. On functions analytic in a half plane, Trans. Amer. Math. Soc. vol. 81, pp. 167-194.

G. N. Watson

1944. The theory of Bessel functions, Cambridge.

G. M. WiNG

1950. The mean convergence of orthogonal series, Amer. J. Math. vol. 72, pp. 792-807.

1951. On the $L_{p}$ theory of Hankel transforms, Pacific J. Math. vol. 1, pp. 313-319.

A. ZYGMUND

1935. Trigonometrical series, Warsaw-Lwow.

1938. On the convergence and summability of power series on the circle of convergence I, Fund. Math. vol. 30, pp. 170-196.

1945. Proof of a theorem of Littlewood and Paley, Bull. Amer. Math. Soc. vol. 51, pp. 439446.

WASHINGTON UNIVERSITY,

ST. Louis, MissourI

UNIVERSITY OF NEBRASKA,

LiNCOLN, NEBRASKA 